TRANSACTIONS OF THE

AMERICAN MATHEMATICAL SOCIETY

Volume 354, Number 7 , Pages 2897-2919

S 0002-9947(02)02994-X

Article electronically published on March 14, 2002

\title{
THE INVERSE PROBLEM \\ OF THE CALCULUS OF VARIATIONS: THE USE OF GEOMETRICAL CALCULUS IN DOUGLAS'S ANALYSIS
}

\author{
W. SARLET, G. THOMPSON, AND G. E. PRINCE
}

\begin{abstract}
The main objective of this paper is to work out a full-scale application of the integrability analysis of the inverse problem of the calculus of variations, as developed in recent papers by Sarlet and Crampin. For this purpose, the celebrated work of Douglas on systems with two degrees of freedom is taken as the reference model. It is shown that the coordinate-free, geometrical calculus used in Sarlet and Crampin's general theoretical developments provides effective tools also to do the practical calculations. The result is not only that all subcases distinguished by Douglas can be given a more intrinsic characterization, but also that in most of the cases, the calculations can be carried out in a more efficient way and often lead to sharper conclusions.
\end{abstract}

\section{INTRODUCTION}

In a number of recent papers, we have revisited the inverse problem of the calculus of variations for second-order ordinary differential equations in its most difficult aspect: that of classifying equations

$$
\ddot{x}^{i}=f^{i}(t, x, \dot{x}) \quad(i=1,2, \ldots, n),
$$

for which a non-singular symmetric multiplier matrix $g_{i j}(t, x, \dot{x})$ exists, such that

$$
g_{i j}\left(\ddot{x}^{j}-f^{j}\right) \equiv \frac{d}{d t}\left(\frac{\partial L}{\partial \dot{x}^{i}}\right)-\frac{\partial L}{\partial x^{i}},
$$

for some function $L$. Such a classification of course has to be achieved by means of conditions on the given data $f^{i}$; and, given the fact that the so-called Helmholtz conditions to be satisfied by $g_{i j}$ involve algebraic as well as partial differential equations, one can expect that addressing the classification question will amount to a systematic integrability analysis of those conditions.

The first author who addressed this integrability aspect of the inverse problem was Douglas [5. His paper, which dealt exclusively with systems with two degrees of freedom $(n=2)$, became notorious, because its complexity for a long time discouraged any further attempt to "solve" the inverse problem in this sense for systems with more than two degrees of freedom. The first novel contribution to the integrability problem perhaps came with the work of Anderson and Thompson 1. Among other things, they were the first who succeeded in generalizing one of

Received by the editors October 25, 2000.

2000 Mathematics Subject Classification. Primary 37J05, 70H03; Secondary 49N45.

Key words and phrases. Lagrangian systems, inverse problem, geometrical calculus. 
the cases in Douglas's classification (Case I) to an arbitrary number of degrees of freedom.

Our own renewed interest in the inverse problem came from the development of an interesting geometrical calculus, first related to autonomous equations (see 7, 8, 9]) and later generalized to time-dependent systems in [14, 2. In 4, it was observed that this calculus was particularly suitable for understanding Douglas's analysis from a geometrical point of view. Indeed, it was shown explicitly how the different cases distinguished by Douglas could be given a coordinate-free interpretation. There were strong indications that the new calculus would lend itself to the application of the old Riquier-Janet theory of formally integrable partial differential equations 111 in a modern coordinate-free way, and this for arbitrary $n$. This challenge was addressed in [13, where the authors gave a fairly complete account of the integrability or passivity conditions, the way they occur in the Riquier approach. The completeness of the analysis is restricted by two conditions, however: firstly, it must be possible to select a so-called proper ordering for the Riquier algorithm; secondly, a certain class of passivity conditions, which in general can be of second order, should not degenerate into conditions of first or zeroth order. The first point was in fact overlooked in [13] and has only recently been clarified [12]. Although this is all one could possibly hope to obtain by this method at such a level of generality, it turns out that this completion of the integrability analysis, when related to Douglas's work for $n=2$, would really bring us to the end of the line in his Case I only (and indeed, this case was also generalized to arbitrary $n$ in [13]). On the other hand, one should not be led to believe then that no progress was made for the other cases. For example, our geometrical approach very often succeeds in reproducing in one fell swoop expressions which Douglas had to compute in coordinates over and over again for each subcase. Further evidence of the value of the method was given in [3], where also Douglas's Case IIa1 was generalized to arbitrary $n$.

With the present paper, we want to pay tribute to the celebrated work of Douglas, by reinvestigating the case $n=2$ with our more intrinsic version of the Riquier algorithm. Unlike other modern approaches to the subject, in particular the recent contributions by Grifone and Muzsnay [6, 10], which are based on the SpencerGoldschmidt theory of formal integrability, our geometrical approach is based on the same underlying methodology as the analytical work of Douglas. We are therefore in a favourable position to streamline his results.

Let us now briefly sketch the basics of our geometrical calculus. Consider a bundle $\pi: E \rightarrow \mathbb{R}$ and its first jet bundle $J^{1} \pi$ with, in particular, the projection $\pi_{1}^{0}: J^{1} \pi \rightarrow E$. The most specific feature of our calculus is that it deals with tensor fields and operators, defined, not on the manifold $J^{1} \pi$, but rather along the projection $\pi_{1}^{0}$. The non-singular symmetric matrix $g_{i j}$ we are looking for in the inverse problem is to be regarded as providing the components of a symmetric type $(0,2)$ tensor field $g$ along $\pi_{1}^{0}$. As reported in many of the above cited papers, a geometrical formulation of the Helmholtz conditions to be satisfied by $g$ reads as follows:

$$
\begin{aligned}
\nabla g & =0, \\
\mathrm{D}_{X_{i}}^{V} g\left(X_{j}, X_{k}\right) & =\mathrm{D}_{X_{k}}^{V} g\left(X_{j}, X_{i}\right), \\
\mathrm{D}_{X_{i}}^{H} g\left(X_{j}, X_{k}\right) & =\mathrm{D}_{X_{k}}^{H} g\left(X_{j}, X_{i}\right), \\
g\left(\Phi\left(X_{i}\right), X_{j}\right) & =g\left(X_{i}, \Phi\left(X_{j}\right)\right) .
\end{aligned}
$$


Here, $\Phi$ is the Jacobi endomorphism, a type $(1,1)$ tensor field along $\pi_{1}^{0}$, coming from a given second-order system

$$
\Gamma=\frac{\partial}{\partial t}+\dot{x}^{i} \frac{\partial}{\partial x^{i}}+f^{i}(t, x, \dot{x}) \frac{\partial}{\partial \dot{x}^{i}}
$$

on $J^{1} \pi$. The components of $\Phi$ are

$$
\Phi_{j}^{i}=-\frac{\partial f^{i}}{\partial x^{j}}-\Gamma_{k}^{i} \Gamma_{j}^{k}-\Gamma\left(\Gamma_{j}^{i}\right),
$$

where

$$
\Gamma_{k}^{i}=-\frac{1}{2} \frac{\partial f^{i}}{\partial \dot{x}^{k}}
$$

are the connection coefficients of the non-linear connection associated to $\Gamma$. The operators $\nabla, \mathrm{D}_{X_{i}}^{V}$ and $\mathrm{D}_{X_{i}}^{H}$ are degree zero derivations acting on tensor fields along $\pi_{1}^{0}$. We shall list important relations and properties concerning these operations when needed, but in large parts of the calculations in the present paper, they will in fact be acting on functions $F$ on $J^{1} \pi$, and their action then is simply that of a vector field: we have

$$
\nabla F=\Gamma(F), \quad \mathrm{D}_{X}^{V} F=X^{V}(F), \quad \mathrm{D}_{X}^{H} F=X^{H}(F),
$$

where $X$ is a vector field along $\pi_{1}^{0}$, and $X^{V}$ and $X^{H}$ are vector fields on $J^{1} \pi$, with the following coordinate representation:

$$
X=X^{i}(x, \dot{x}) \frac{\partial}{\partial x^{i}}, \quad X^{V}=X^{i} \frac{\partial}{\partial \dot{x}^{i}}, \quad X^{H}=X^{i}\left(\frac{\partial}{\partial x^{i}}-\Gamma_{i}^{j} \frac{\partial}{\partial \dot{x}^{j}}\right) .
$$

As the representation of $X$ indicates, all vector fields along $\pi_{1}^{0}$ which enter the calculations in the present context take their values in the vertical subbundle of $T E$ (and in fact also all tensor fields under consideration act on such vectors only). As a result, although the set-up is about time-dependent systems, the formulas of the geometrical calculus we need are rather those of the autonomous theory, and derivatives with respect to time only occur in the dynamical covariant derivative $\nabla$. To complete the picture, it suffices to recall how the derivations under consideration act on the local bases of vector fields and 1-forms along $\pi_{1}^{0}$. We have

$$
\begin{aligned}
\nabla\left(\frac{\partial}{\partial x^{i}}\right) & =\Gamma_{i}^{j} \frac{\partial}{\partial x^{j}}, \quad \mathrm{D}_{X}^{V}\left(\frac{\partial}{\partial x^{i}}\right)=0, \quad \mathrm{D}_{X}^{H}\left(\frac{\partial}{\partial x^{i}}\right)=X^{V}\left(\Gamma_{i}^{j}\right) \frac{\partial}{\partial x^{j}}, \\
\nabla\left(d x^{i}\right) & =-\Gamma_{j}^{i} d x^{j}, \quad \mathrm{D}_{X}^{V}\left(d x^{i}\right)=0, \quad \mathrm{D}_{X}^{H}\left(d x^{i}\right)=-X^{V}\left(\Gamma_{j}^{i}\right) d x^{j} .
\end{aligned}
$$

Now, to be a bit more precise, the most succinct version of the Helmholtz conditions is actually a subset of the set of conditions listed above, namely the conditions (1.3), (1.4) and (1.6). But the extra condition (1.5) is also a necessary one: it is the first integrability requirement which has to be added on as a result of comparing prolongations of (1.3) and (1.4), knowing the property

$$
\left[\nabla, \mathrm{D}_{X}^{V}\right]=\mathrm{D}_{\nabla X}^{V}-\mathrm{D}_{X}^{H} .
$$

Other obvious necessary requirements, which follow from compatibility between (1.3) and (1.6), are the algebraic conditions

$$
g\left(\nabla^{k} \Phi\left(X_{i}\right), X_{j}\right)=g\left(X_{i}, \nabla^{k} \Phi\left(X_{j}\right)\right), \quad \forall k \geq 1 .
$$


There is another infinite hierarchy of algebraic conditions on $g$, involving the curvature $R$ of the non-linear connection (see e.g. [13]), but it will be less important here, as it is void for $n=2$.

The idea is first to exhaust all the information coming from the algebraic requirements on $g$ (these might, in fact, simply reduce the dimension of the solution space to zero). Once we have done that, the Riquier algorithm requires writing the closure conditions (1.4 1.5) in a systematic way in order to obtain a system of equations in orthonomic form. This involves selecting an ordering for the dependent variables and their derivatives, and will determine, for each equation of type (1.4) or (1.5), which term is chosen for the left-hand side (and is then called a principal derivative) and which are the terms on the right-hand side (called parametric derivatives). At this point, the concept of a proper ordering, as introduced in 12, becomes important. Essentially, the ordering will be proper if whenever two left-hand sides of (1.4) refer to the same component of $g, \mathrm{D}_{X_{i}}^{V} g\left(X_{j}, X_{k}\right)$ and $\mathrm{D}_{X_{l}}^{V} g\left(X_{j}, X_{k}\right)$ for example, the corresponding right-hand sides involve derivatives with respect to the same argument, i.e. they are for example $\mathrm{D}_{X_{j}}^{V} g\left(X_{i}, X_{k}\right)$ and $\mathrm{D}_{X_{j}}^{V} g\left(X_{l}, X_{k}\right)$. If the ordering is proper, the next compatibility requirements come only from comparing $\mathrm{D}^{H}$-prolongations of (1.4) with $\mathrm{D}^{V}$-prolongations of (1.5). They are what we shall call $\mathcal{A}$-conditions, and read:

$$
\begin{aligned}
& \left(\mathcal{A}\left(X_{i}, X_{j}\right) g\right)\left(X_{k}, X_{l}\right) \\
& \quad:=\left(\left(\mathrm{D}_{X_{i}}^{V} \mathrm{D}_{X_{j}}^{H}-\mathrm{D}_{X_{j}}^{V} \mathrm{D}_{X_{i}}^{H}-\mathrm{D}_{\mathrm{D}_{X_{i}}^{H} X_{j}}^{H}+\mathrm{D}_{\mathrm{D}_{X_{j}}^{H} X_{i}}^{H}\right) g\right)\left(X_{k}, X_{l}\right) \\
& \quad+g\left(\theta\left(X_{k}, X_{l}\right) X_{i}, X_{j}\right)-g\left(X_{i}, \theta\left(X_{k}, X_{l}\right) X_{j}\right)=0
\end{aligned}
$$

(see the definition (1.14) for the meaning of the tensor field $\theta$ ). Clearly, these are, in principle, second-order partial differential equations for $g$. It was further shown in [13, 12 that for a proper ordering, provided there is no degeneracy in the conditions (1.13), the process of generating integrability conditions stops right there, which is a quite striking result. For example, any attempt to confront, say, $\mathrm{D}^{V}$-prolongations of the conditions (1.13) with second-order $\mathrm{D}^{V}$-prolongations of (1.5) and $\mathrm{D}^{V} \mathrm{D}^{H}$-prolongations of (1.4), can never give rise to new integrability conditions. When some of the entries of the matrix $g_{i j}$ are zero as a result of algebraic restrictions, however, some or all of the $\mathcal{A}$-conditions will 'degenerate' into first-order equations (or even algebraic ones). The search for further integrability conditions then becomes a different story, as prolongations of the conditions (1.13) then should be compared with first-order prolongations of the conditions (1.3.1.5), so the proof that the procedure terminates breaks down.

When $\Phi$ is a multiple of the identity, there are no algebraic restrictions on $g$; that corresponds to Case I in Douglas's classification and explains why we were able to extend this case to arbitrary dimensions. For all other cases, unfortunately, one is bound to continue the integrability analysis case by case. Nevertheless, our approach enables us to improve significantly on Douglas. Not only is it coordinatefree and valid for arbitrary $n$, but it also tells us where to start the next step in the analysis in each case: the $\mathcal{A}$-conditions, for a proper ordering, cover in a uniform way a large number of very different-looking conditions which Douglas had to compute separately for each case. In fact, there is even one exception to the point made above about the general proof breaking down: indeed, the proof that compatibility between the $\mathcal{A}$-conditions and the equation $\nabla g=0$ is identically satisfied remains 
valid even when the $\mathcal{A}$-conditions degenerate. This is so because the right-hand side of the equation for $\nabla g$ is simply zero (so that it makes no difference whether one takes first- or second-order prolongations), and the fact that no extra vector argument is introduced when taking $\nabla$ prolongations. This observation alone covers numerous situations where Douglas computes compatibility conditions with the $(\partial / \partial t)$-equation for individual components of $g$, only to find in each case that the condition is identically satisfied. There are still other aspects of our $\mathcal{A}$-conditions (1.13) which call for an explicit verification in particular situations. For example, as already reported in earlier work, in Case I, for every condition of type (1.13) we can write down, Douglas would be led to compute two second-order conditions and would conclude that they happen to coincide.

Part of the purpose of the present paper is to illustrate the practical value and utility of the $\mathcal{A}$-conditions in a number of different situations where they degenerate. The only way to do that convincingly is to go back to systems with two degrees of freedom and to show explicitly how we can recover and improve upon results obtained by Douglas in each of the main classes of his classification. Needless to say, this will involve some quite tedious calculations. For a brief summary of the classification scheme of Douglas, we refer to 4. More details will be given as we proceed with the present analysis. We set the stage in the next section, where the differential conditions (1.31.5) and (1.13) are made concrete with respect to an appropriate local basis of vector fields along $\pi_{1}^{0}$, and already a few of the points raised above are verified explicitly. In Section 3, we show how our method works in a number of different subcases with a diagonalizable $\Phi$ and thereby confirms or sharpens the results of Douglas. We do the same in Section 4 for the case that $\Phi$ is not diagonalizable (Case IIb of Douglas). Each of the cases is illustrated by an explicit example in the last section.

One point remains to be explained here: the algebraic part of the $\mathcal{A}$-conditions (1.13) contains a type $(1,3)$ tensor field $\theta$ along $\pi_{1}^{0}$, which is defined as follows:

$$
\theta\left(X_{k}, X_{l}\right) X_{i}:=\left(\mathrm{D}_{X_{k}}^{V} \mathrm{D}_{X_{l}}^{H}-\mathrm{D}_{X_{l}}^{H} \mathrm{D}_{X_{k}}^{V}-\mathrm{D}_{\mathrm{D}_{X_{k}}^{H} X_{l}}^{H}+\mathrm{D}_{\mathrm{D}_{X_{l}}^{H} X_{k}}^{V}\right) X_{i} .
$$

Explicit calculations, when they involve $\theta$, can require some ingenuity, as one often has to make use of the property that $\theta$ is symmetric in all its arguments. As a matter of fact, with respect to a local basis of coordinate vector fields and forms, the components of $\theta$ are proportional to $\partial^{3} f^{i} / \partial \dot{x}^{j} \partial \dot{x}^{k} \partial \dot{x}^{l}$.

\section{The DifFERENTIAL CONDITIONS FOR $n=2$}

In view of the algebraic condition (1.6) which expresses the symmetry of the product of two matrices, one being the unknown $g$, the other one the given $\Phi$, it is natural that a classification of all possible cases will be dictated by the different real Jordan normal form structure that the given $\Phi$ can have. The best way then of writing down explicit expressions for the differential conditions on $g$ will be with respect to a local frame of vector fields along $\pi_{1}^{0}$, maximally adapted to the structure of $\Phi$ (eigenvectors, for example). So, let $\left(X_{i}\right)_{i=1,2}$ be such a frame, to be specified later on for each case separately, and let $\left(\theta^{j}\right)_{j=1,2}$ be the dual coframe of 1-forms. We introduce various structure functions to express covariant derivatives of these elements with respect to the same basis. In general we will put (the summation 
convention is in use unless otherwise specified)

$$
\nabla X_{i}=\nu_{i}^{j} X_{j}, \quad \mathrm{D}_{X_{i}}^{V} X_{j}=\tau_{i j}^{k} X_{k}, \quad \mathrm{D}_{X_{i}}^{H} X_{j}=\mu_{i j}^{k} X_{k} .
$$

The functions $\nu_{i}^{j}, \tau_{i j}^{k}$ and $\mu_{i j}^{k}$ are to be regarded as known functions, and there are relations among them, coming from properties of the derivations such as (1.11). By duality, we likewise have

$$
\nabla \theta^{i}=-\nu_{j}^{i} \theta^{j}, \quad \mathrm{D}_{X_{i}}^{V} \theta^{j}=-\tau_{i k}^{j} \theta^{k}, \quad \mathrm{D}_{X_{i}}^{H} \theta^{j}=-\mu_{i k}^{j} \theta^{k} .
$$

With respect to the selected coframe, the most general expression for a symmetric type $(0,2)$ tensor field $g$ along $\pi_{1}^{0}$ reads

$$
g=\rho_{A} g^{A},
$$

where

$$
g^{1}=\theta^{1} \otimes \theta^{1}, \quad g^{2}=\theta^{2} \otimes \theta^{2}, \quad g^{3}=\theta^{1} \otimes \theta^{2}+\theta^{2} \otimes \theta^{1} .
$$

So, a capital latin index will run from 1 to 3 , and we will also write $\rho=\rho_{3}$ for the off-diagonal component of $g$.

It will also be advantageous to make temporary use of structure functions which relate directly to the basis $\left\{g^{A}\right\}$ of symmetric $(0,2)$ tensors. We put

$$
\mathrm{D}_{X_{i}}^{V} g^{A}=v_{i B}^{A} g^{B}, \quad \mathrm{D}_{X_{i}}^{H} g^{A}=\kappa_{i B}^{A} g^{B},
$$

as a means of easing the writing. One easily verifies from the defining relations that the following identities hold (written down in the format of $3 \times 3$ matrices, with $i=1$ or 2$)$ :

$$
\left(v_{i B}^{A}\right)=-\left(\begin{array}{ccc}
2 \tau_{i 1}^{1} & 0 & \tau_{i 2}^{1} \\
0 & 2 \tau_{i 2}^{2} & \tau_{i 1}^{2} \\
2 \tau_{i 1}^{2} & 2 \tau_{i 2}^{1} & \tau_{i 1}^{1}+\tau_{i 2}^{2}
\end{array}\right), \quad\left(\kappa_{i B}^{A}\right)=-\left(\begin{array}{ccc}
2 \mu_{i 1}^{1} & 0 & \mu_{i 2}^{1} \\
0 & 2 \mu_{i 2}^{2} & \mu_{i 1}^{2} \\
2 \mu_{i 1}^{2} & 2 \mu_{i 2}^{1} & \mu_{i 1}^{1}+\mu_{i 2}^{2}
\end{array}\right) .
$$

We now set up some master expressions for the differential conditions (1.4), (1.5) and (1.13), which will then have to be adapted to each of the different situations coming from the algebraic conditions (1.6) (or possibly even (1.12)). There is no need to do that for equation (1.3) at the moment, since it hardly plays a role in the integrability discussion, for reasons already indicated. With $n=2$, we have two conditions in the grouping (1.4) (and likewise for (1.5)), namely $\mathrm{D}_{X_{1}}^{V} g\left(X_{1}, X_{2}\right)=$ $\mathrm{D}_{X_{2}}^{V} g\left(X_{1}, X_{1}\right)$ and $\mathrm{D}_{X_{1}}^{V} g\left(X_{2}, X_{2}\right)=\mathrm{D}_{X_{2}}^{V} g\left(X_{2}, X_{1}\right)$. The computations are now straightforward: since all operations involved are degree zero derivations, we write, for example,

$$
\mathrm{D}_{X_{j}}^{V} g=\left(\mathrm{D}_{X_{j}}^{V} \rho_{B}\right) g^{B}+\rho_{A} v_{j B}^{A} g^{B},
$$

and then evaluate such tensors on the appropriate arguments. This way, the socalled closure conditions (1.4) and (1.5) become

$$
\begin{aligned}
& \mathrm{D}_{X_{2}}^{V} \rho_{1}=\mathrm{D}_{X_{1}}^{V} \rho+\rho_{A}\left(v_{13}^{A}-v_{21}^{A}\right), \\
& \mathrm{D}_{X_{1}}^{V} \rho_{2}=\mathrm{D}_{X_{2}}^{V} \rho+\rho_{A}\left(v_{23}^{A}-v_{12}^{A}\right), \\
& \mathrm{D}_{X_{2}}^{H} \rho_{1}=\mathrm{D}_{X_{1}}^{H} \rho+\rho_{A}\left(\kappa_{13}^{A}-\kappa_{21}^{A}\right), \\
& \mathrm{D}_{X_{1}}^{H} \rho_{2}=\mathrm{D}_{X_{2}}^{H} \rho+\rho_{A}\left(\kappa_{23}^{A}-\kappa_{12}^{A}\right) .
\end{aligned}
$$


As long as the closure conditions can be written in the above format, there is no danger about the ordering: the ordering is proper simply because the two vertical derivatives do not involve the same $\rho_{i}$. The situation may be different if algebraic restrictions force one or both of the $\rho_{i}$ to be zero.

Concerning (1.13), since the $\mathcal{A}$-operator is skew-symmetric, the only (symmetric) tensor field to compute here is $\mathcal{A}\left(X_{1}, X_{2}\right) g$. Leaving the algebraic terms involving the $\theta$ tensor (1.14) unspecified at the moment, one obtains

$$
\begin{aligned}
& \mathcal{A}\left(X_{1}, X_{2}\right) g=\left[\left(\mathrm{D}_{X_{1}}^{V} \mathrm{D}_{X_{2}}^{H}-\mathrm{D}_{X_{2}}^{V} \mathrm{D}_{X_{1}}^{H}\right)\left(\rho_{B}\right)+v_{1 B}^{A} \mathrm{D}_{X_{2}}^{H} \rho_{A}-v_{2 B}^{A} \mathrm{D}_{X_{1}}^{H} \rho_{A}\right. \\
& \quad+\kappa_{2 B}^{A} \mathrm{D}_{X_{1}}^{V} \rho_{A}-\kappa_{1 B}^{A} \mathrm{D}_{X_{2}}^{V} \rho_{A}+\rho_{A}\left(\mathrm{D}_{X_{1}}^{V} \kappa_{2 B}^{A}-\mathrm{D}_{X_{2}}^{V} \kappa_{1 B}^{A}\right) \\
& \left.\quad+\rho_{A}\left(\kappa_{2 C}^{A} v_{1 B}^{C}-\kappa_{1 C}^{A} v_{2 B}^{C}\right)-\left(\tau_{12}^{k}-\tau_{21}^{k}\right) \mathrm{D}_{X_{k}}^{H} \rho_{B}-\left(\tau_{12}^{k}-\tau_{21}^{k}\right) \rho_{A} \kappa_{k B}^{A}\right] g^{B} \\
& \quad+\rho_{B} g^{B}\left(\theta(\cdot, \cdot) X_{1}, X_{2}\right)-\rho_{B} g^{B}\left(X_{1}, \theta(\cdot, \cdot) X_{2}\right) .
\end{aligned}
$$

One then has to decide which components of this symmetric tensor field are relevant. Recall that all derivatives of principal derivatives are principal also. The rule of thumb in this matter then simply is: for a proper ordering, the $\mathcal{A}$-conditions to be considered are those for which the second-order derivative terms are not principal derivatives. So, as long as $\rho_{1}$ and $\rho_{2}$ are not zero, there is just a single $\mathcal{A}$-condition, namely

$$
\left(\mathcal{A}\left(X_{1}, X_{2}\right) g\right)\left(X_{1}, X_{2}\right)=0 .
$$

It will clearly produce a second-order partial differential equation for $\rho$ (excluding for the moment the case that $\rho$ is zero). This is in a way a rather challenging claim when we compare it to the calculations in [5], especially since the direct calculations of [5] also make perfect sense in our more geometrical set-up. Indeed, suppose we look directly at equations $(2.7 \cdot 2.10$ ) and enquire about integrability conditions. The left-hand sides are after all just vector fields acting on functions, so we can proceed directly via Frobenius's theorem. Clearly then, there are two conditions to be investigated, one coming from the commutator $\left[X_{2}^{V}, X_{2}^{H}\right]$ acting on the function $\rho_{1}$ and the other one coming from $\left[X_{1}^{V}, X_{1}^{H}\right]$ acting on $\rho_{2}$. How does this match the claim that there is just a single $\mathcal{A}$-condition to be verified? The first point to observe from the structure of equations (2.7), (2.9), respectively (2.8), (2.10), is that both computations will give rise to a second-order equation for $\rho$. In an appendix, we briefly sketch how one can proceed to show that these two equations are equivalent, i.e., they are the same after all substitutions of parametric for principal derivatives have been carried out. We further leave it as an exercise for the reader to verify that they are also equivalent in the same sense to the single second-order equation for $\rho$ coming from (2.12). This of course requires computing (2.12) in full detail, but the reader will find enough indications of how to do this in the next section, where we will effectively compute (2.12) in a situation where it "degenerates" into a lower-order condition because of the fact that $\rho$ is zero. Finally, a second interesting exercise is to verify explicitly another aspect of our claim. Indeed, our theory predicts that the diagonal components of the tensor field $\mathcal{A}\left(X_{1}, X_{2}\right) g$ do not give rise to new restrictions here; that is to say, $\left(\mathcal{A}\left(X_{1}, X_{2}\right) g\right)\left(X_{k}, X_{k}\right) \equiv 0(k=1,2)$ when substituting parametric for principal derivatives. Again, it would not be fruitful to verify this here, but Section 4 will show the way to do it, because we will then be in a situation where precisely these diagonal components are the $\mathcal{A}$-conditions that matter. 


\section{THE CASES WHERE $\Phi$ IS DIAGONALIZABLE}

As explained in [4], the main classification scheme of Douglas comes from looking at the dependence (or independence) of the matrices $\Phi, \nabla \Phi, \nabla^{2} \Phi$ and the identity $I$. Inside each of his three main cases (we exclude his Case IV from our considerations because then the system can never be variational), further subdivisions have to do with properties of the eigenvalues of $\Phi$, or in other words with the nature of the Jordan normal form of $\Phi$. We, on the other hand, will base our investigations here directly on the latter aspect and will arrive this way at subcases of the three different main classes of Douglas.

Assume that $\Phi$ is diagonalizable (with real eigenvalues for simplicity). If the eigenvalues are equal, we have $\Phi=\lambda I$, which is Case I of Douglas, the case that was extensively discussed (for general $n$ ) in [13] and was found to be variational by several authors. Assume therefore that the two eigenvalues $\lambda_{i}$ are distinct. We may then be in Case IIa of Douglas or in Case III (cf. [4]). Let $X_{i}$ be vector fields along $\pi_{1}^{0}$ which each span one of the 1 -dimensional eigendistributions. This is the local basis of vector fields along $\pi_{1}^{0}$ with respect to which we do the calculations now. There is still some scale freedom, which we will exploit later on.

The first algebraic condition (1.6) implies that $g$ will be diagonal (with respect to the dual basis $\theta^{i}$ which here are eigenforms of $\left.\Phi\right)$ :

$$
g=\rho_{1} \theta^{1} \otimes \theta^{1}+\rho_{2} \theta^{2} \otimes \theta^{2}
$$

With $\rho=0$, the closure conditions become

$$
\begin{aligned}
& \mathrm{D}_{X_{2}}^{V} \rho_{1}=\rho_{k}\left(v_{13}^{k}-v_{21}^{k}\right)=\left(2 \tau_{21}^{1}-\tau_{12}^{1}\right) \rho_{1}-\tau_{11}^{2} \rho_{2}, \\
& \mathrm{D}_{X_{1}}^{V} \rho_{2}=\rho_{k}\left(v_{23}^{k}-v_{12}^{k}\right)=\left(2 \tau_{12}^{2}-\tau_{21}^{2}\right) \rho_{2}-\tau_{22}^{1} \rho_{1}, \\
& \mathrm{D}_{X_{2}}^{H} \rho_{1}=\rho_{k}\left(\kappa_{13}^{k}-\kappa_{21}^{k}\right)=\left(2 \mu_{21}^{1}-\mu_{12}^{1}\right) \rho_{1}-\mu_{11}^{2} \rho_{2}, \\
& \mathrm{D}_{X_{1}}^{H} \rho_{2}=\rho_{k}\left(\kappa_{23}^{k}-\kappa_{12}^{k}\right)=\left(2 \mu_{12}^{2}-\mu_{21}^{2}\right) \rho_{2}-\mu_{22}^{1} \rho_{1} .
\end{aligned}
$$

We now proceed to compute the $\mathcal{A}$-condition (2.12) explicitly (it is not much harder to do it without the simplification $\rho=0$ for comparison with the secondorder conditions derived in the Appendix). We shall split the expression in different parts for a more transparent view of the way to proceed. First, we compute the derivative terms in (1.13) (with $i=k=1, j=l=2$ ). In other words, we take the first three lines of the right-hand side of (2.11) and evaluate them on the pair $\left(X_{1}, X_{2}\right)$. It is clear then that in the sum over $B$, only the term for $B=3$ survives. We denote this part of the expression by $\left(A\left(X_{1}, X_{2}\right) g\right)\left(X_{1}, X_{2}\right)$. Needless to say, derivatives of the $\rho_{i}$ which are left-hand sides in the closure conditions (3.2.3.5) have to be replaced by the corresponding right-hand sides. The result is

$$
\begin{aligned}
& \left(A\left(X_{1}, X_{2}\right) g\right)\left(X_{1}, X_{2}\right)=v_{13}^{2} \mathrm{D}_{X_{2}}^{H} \rho_{2}-v_{23}^{1} \mathrm{D}_{X_{1}}^{H} \rho_{1}+\kappa_{23}^{1} \mathrm{D}_{X_{1}}^{V} \rho_{1}-\kappa_{13}^{2} \mathrm{D}_{X_{2}}^{V} \rho_{2} \\
& \quad+\rho_{i}\left[v_{13}^{1}\left(\kappa_{13}^{i}-\kappa_{21}^{i}\right)-v_{23}^{2}\left(\kappa_{23}^{i}-\kappa_{12}^{i}\right)-\kappa_{13}^{1}\left(v_{13}^{i}-v_{21}^{i}\right)+\kappa_{23}^{2}\left(v_{23}^{i}-v_{12}^{i}\right)\right. \\
& \left.\quad+\mathrm{D}_{X_{1}}^{V} \kappa_{23}^{i}-\mathrm{D}_{X_{2}}^{V} \kappa_{13}^{i}+\kappa_{2 C}^{i} v_{13}^{C}-\kappa_{1 C}^{i} v_{23}^{C}-\left(\tau_{12}^{k}-\tau_{21}^{k}\right) \kappa_{k 3}^{i}\right] .
\end{aligned}
$$


Eventually, one has to express everything in terms of the $\tau_{i j}^{k}$ and $\mu_{i j}^{k}$ via (2.6). The result then reads

$$
\begin{aligned}
& \left(A\left(X_{1}, X_{2}\right) g\right)\left(X_{1}, X_{2}\right)=-\tau_{11}^{2} \mathrm{D}_{X_{2}}^{H} \rho_{2}+\tau_{22}^{1} \mathrm{D}_{X_{1}}^{H} \rho_{1}-\mu_{22}^{1} \mathrm{D}_{X_{1}}^{V} \rho_{1}+\mu_{11}^{2} \mathrm{D}_{X_{2}}^{V} \rho_{2} \\
& \quad+\rho_{1}\left[\mathrm{D}_{X_{2}}^{V} \mu_{12}^{1}-\mathrm{D}_{X_{1}}^{V} \mu_{22}^{1}+\mu_{22}^{1}\left(\tau_{11}^{1}+2 \tau_{12}^{2}-2 \tau_{21}^{2}\right)+\tau_{22}^{1}\left(\mu_{21}^{2}-2 \mu_{11}^{1}\right)\right. \\
& \left.\quad-\mu_{12}^{1}\left(\tau_{22}^{2}-\tau_{12}^{1}\right)\right]-\rho_{2}[1 \leftrightarrow 2]
\end{aligned}
$$

where the coefficient of $-\rho_{2}$ is obtained from that of $\rho_{1}$ by interchanging all indices 1 and 2. To this we still have to add the terms

$$
\begin{aligned}
& g\left(\theta\left(X_{1}, X_{2}\right) X_{1}, X_{2}\right)-g\left(X_{1}, \theta\left(X_{1}, X_{2}\right) X_{2}\right) \\
& \quad=\rho_{2}\left\langle\theta\left(X_{1}, X_{2}\right) X_{1}, \theta^{2}\right\rangle-\rho_{1}\left\langle\theta\left(X_{1}, X_{2}\right) X_{2}, \theta^{1}\right\rangle .
\end{aligned}
$$

The computation of the first term proceeds as follows. From the defining relation (1.14), we have

$$
\begin{aligned}
\theta\left(X_{1},\right. & \left.X_{2}\right) X_{1}=\left(\mathrm{D}_{X_{1}}^{V} \mathrm{D}_{X_{2}}^{H}-\mathrm{D}_{X_{2}}^{H} \mathrm{D}_{X_{1}}^{V}-\mathrm{D}_{\mathrm{D}_{X_{1}} X_{2}}^{H}+\mathrm{D}_{\mathrm{D}_{X_{2}} X_{1}}^{V}\right) X_{1} \\
= & \mathrm{D}_{X_{1}}^{V}\left(\mu_{21}^{1} X_{1}+\mu_{21}^{2} X_{2}\right)-\mathrm{D}_{X_{2}}^{H}\left(\tau_{11}^{1} X_{1}+\tau_{11}^{2} X_{2}\right)-\tau_{12}^{1}\left(\mu_{11}^{1} X_{1}+\mu_{11}^{2} X_{2}\right) \\
& -\tau_{12}^{2}\left(\mu_{21}^{1} X_{1}+\mu_{21}^{2} X_{2}\right)+\mu_{21}^{1}\left(\tau_{11}^{1} X_{1}+\tau_{11}^{2} X_{2}\right)+\mu_{21}^{2}\left(\tau_{21}^{1} X_{1}+\tau_{21}^{2} X_{2}\right),
\end{aligned}
$$

from which it follows that

$$
\begin{aligned}
\left\langle\theta\left(X_{1}, X_{2}\right) X_{1}, \theta^{2}\right\rangle= & \mu_{21}^{1} \tau_{11}^{2}+\mathrm{D}_{X_{1}}^{V} \mu_{21}^{2}-\tau_{11}^{1} \mu_{21}^{2}-\mathrm{D}_{X_{2}}^{H} \tau_{11}^{2} \\
& -\tau_{11}^{2} \mu_{22}^{2}-\tau_{12}^{1} \mu_{11}^{2}+\mu_{21}^{1} \tau_{11}^{2}+\mu_{21}^{2} \tau_{21}^{2} .
\end{aligned}
$$

This expression has to be added to the coefficient of $\rho_{2}$ in (3.7), which gives rise to some simplifications. For the other term in (3.8), we can make good use of the symmetry properties of the $\theta$ tensor. Indeed, we know for example that

$$
\theta\left(X_{1}, X_{2}\right) X_{2}=\theta\left(X_{2}, X_{1}\right) X_{2},
$$

and since the coefficients of $\rho_{1}$ and $-\rho_{2}$ in (3.7) can be mapped into one another by interchanging 1 and 2 everywhere, it is clear that we had better use the right-hand side of the above equality to compute the second term in (3.8).

The final equation we obtain this way, reads as follows:

$$
\begin{aligned}
0= & \tau_{22}^{1} \mathrm{D}_{X_{1}}^{H} \rho_{1}-\mu_{22}^{1} \mathrm{D}_{X_{1}}^{V} \rho_{1}-\tau_{11}^{2} \mathrm{D}_{X_{2}}^{H} \rho_{2}+\mu_{11}^{2} \mathrm{D}_{X_{2}}^{V} \rho_{2} \\
& +\rho_{1}\left[\mu_{22}^{1}\left(2 \tau_{12}^{2}-\tau_{21}^{2}+\tau_{11}^{1}\right)-\tau_{22}^{1}\left(2 \mu_{12}^{2}-\mu_{21}^{2}+\mu_{11}^{1}\right)+\mathrm{D}_{X_{1}}^{H} \tau_{22}^{1}-\mathrm{D}_{X_{1}}^{V} \mu_{22}^{1}\right] \\
& -\rho_{2}\left[\mu_{11}^{2}\left(2 \tau_{21}^{1}-\tau_{12}^{1}+\tau_{22}^{2}\right)-\tau_{11}^{2}\left(2 \mu_{21}^{1}-\mu_{12}^{1}+\mu_{22}^{2}\right)+\mathrm{D}_{X_{2}}^{H} \tau_{11}^{2}-\mathrm{D}_{X_{2}}^{V} \mu_{11}^{2}\right]
\end{aligned}
$$

This is the explicit expression for the only $\mathcal{A}$-condition which has to be imposed in all situations where $\Phi$ is diagonalizable with distinct eigenvalues, and it is at most a first-order partial differential equation. Hence, unless (3.11) happens to be identically satisfied, we know that further integrability analysis with the closure conditions will be necessary. We must not forget also that further algebraic restrictions may have to be imposed, coming from the hierarchy (1.12).

In agreement with the objectives set out in the Introduction, we now focus on a number of different subcases of Douglas which have in common that $\Phi$ is diagonalizable with distinct eigenvalues. Assume first that $[\nabla \Phi, \Phi]=0$. For $n=2$ this is equivalent to saying that $\nabla \Phi=a \Phi+b I$ for some functions $a, b \in C^{\infty}\left(J^{1} \pi\right)$, so there are no further restrictions coming from the hierarchy (1.12), and we are in Case IIa of Douglas. It follows from the extra assumption that the eigenspaces of 
$\Phi$ are $\nabla$-invariant, so that $\nabla X_{i} \propto X_{i}, i=1,2$, and since one can always rescale the $X_{i}$ in such a way that the functions $\nu_{i}^{i}$ in (2.1) are zero, we can assume here that $\nabla X_{i}=0$. It then follows from the general commutator property (1.11) that

$$
\mu_{i j}^{k}=-\nabla \tau_{i j}^{k}
$$

Moreover, the equation $\nabla g=0$, which we have not specified so far, in this situation simply becomes

$$
\nabla \rho_{1}=\nabla \rho_{2}=0
$$

i.e. the $\rho_{i}$ must be first integrals.

For completeness, we first recall Case IIa1, called by Douglas the 'separated case'. It is the case where the closure conditions 3.2+3.5 decouple. In view of $(3.12)$, it is obvious that this requires

$$
\tau_{22}^{1}=\tau_{11}^{2}=0
$$

and we immediately see that (3.11) is identically satisfied, so the system is variational. (We observed already in [4] that (3.14) are tensorial properties: they are equivalent to the vanishing of the type $(1,2)$ tensor field $H_{\Phi}$, defined by

$$
H_{\Phi}\left(X_{k}, X_{l}\right):=C_{\Phi}^{V}\left(\Phi X_{k}, X_{l}\right)-\Phi\left(C_{\Phi}^{V}\left(X_{k}, X_{l}\right)\right)
$$

where

$$
C_{\Phi}^{V}\left(X_{k}, X_{l}\right):=\left[\mathrm{D}_{X_{k}}^{V} \Phi, \Phi\right]\left(X_{l}\right) .
$$

In [3, it was shown, via the same type of calculations, that this situation actually has an extension to arbitrary $n$.)

Let us now test the method further on Case IIa2 (the 'semi-separated case'), where the closure conditions only partially decouple, say

$$
\tau_{11}^{2}=0, \quad \text { but } \quad \tau_{22}^{1} \neq 0
$$

This is also a geometrical condition, as it means that only one of the two components of $H_{\Phi}$ (which are not identically zero for the type of $\Phi$ under consideration) vanishes.

A schematic summary of Douglas's results for this case reads as follows: beyond the closure conditions, there are two new integrability conditions which turn out to coincide and give a first-order equation for $\rho_{1}$; the further integrability analysis is reduced to the four equations one then has for $\rho_{1}$; compatibility between the new equation and the $\nabla$ equation is identically satisfied; compatibility between the new equation and the $\mathrm{D}^{V}$ and $\mathrm{D}^{H}$ equations (i.e. the reduced equations (3.2) and (3.4) may result in two new first-order conditions involving the same derivative of $\rho_{1}$; an algebraic discussion then has to be conducted concerning the internal consistency of these two new equations; if they are effectively first-order equations, but then linearly dependent for consistency, all derivatives of $\rho_{1}$ have become principal derivatives and integrability becomes a matter of a certain 1-form being closed.

Let us see how this materialises in our approach to Case IIa2. Beyond the closure conditions, we generate only one new condition, namely the $\mathcal{A}$-condition (3.11), which in view of the assumptions (3.17) reduces to

$$
\begin{aligned}
& \tau_{22}^{1} \mathrm{D}_{X_{1}}^{H} \rho_{1}-\mu_{22}^{1} \mathrm{D}_{X_{1}}^{V} \rho_{1} \\
& \quad=\rho_{1}\left[-\mu_{22}^{1}\left(2 \tau_{12}^{2}-\tau_{21}^{2}+\tau_{11}^{1}\right)+\tau_{22}^{1}\left(2 \mu_{12}^{2}-\mu_{21}^{2}+\mu_{11}^{1}\right)-\mathrm{D}_{X_{1}}^{H} \tau_{22}^{1}+\mathrm{D}_{X_{1}}^{V} \mu_{22}^{1}\right]
\end{aligned}
$$


with the $\mu_{i j}^{k}$ given by (3.12). We know that the new equation will be compatible with the equation $\nabla \rho_{1}=0$, but it is of interest to explore this compatibility in more detail. By the nature of (3.18), we are led to introduce the vector field

$$
Z=\tau_{22}^{1} X_{1}^{H}+\nabla \tau_{22}^{1} X_{1}^{V}
$$

Writing the coefficient of $\rho_{1}$ for shorthand as $b$, so that

$$
b=\nabla \tau_{22}^{1}\left(2 \tau_{12}^{2}-\tau_{21}^{2}+\tau_{11}^{1}\right)-\tau_{22}^{1} \nabla\left(2 \tau_{12}^{2}-\tau_{21}^{2}+\tau_{11}^{1}\right)-\mathrm{D}_{X_{1}}^{H} \tau_{22}^{1}-\mathrm{D}_{X_{1}}^{V} \nabla \tau_{22}^{1},
$$

and putting $\varrho_{1}=\ln \rho_{1}$, (3.18) becomes

$$
Z\left(\varrho_{1}\right)=b .
$$

Recall that, in vector field notations, the other equations to be satisfied by $\varrho_{1}$ are

$$
\Gamma\left(\varrho_{1}\right)=0, \quad X_{2}^{V}\left(\varrho_{1}\right)=\left(2 \tau_{21}^{1}-\tau_{12}^{1}\right), \quad X_{2}^{H}\left(\varrho_{1}\right)=-\Gamma\left(2 \tau_{21}^{1}-\tau_{12}^{1}\right) .
$$

Now we reveal some more properties of the known structure functions $\tau_{i j}^{k}$.

Similar to the relation (1.11), there is a corresponding but more complicated commutator property for the operators $\nabla$ and $\mathrm{D}_{X_{i}}^{H}$; for its action on the module of vector fields along $\pi_{1}^{0}$ we have

$$
\left[\nabla, \mathrm{D}_{X_{i}}^{H}\right] X_{j}=\mathrm{D}_{\nabla X_{i}}^{H} X_{j}+\mathrm{D}_{\Phi X_{i}}^{V} X_{j}-\left(\mathrm{D}_{X_{j}}^{V} \Phi\right)\left(X_{i}\right)-R\left(X_{i}, X_{j}\right),
$$

where $R$ is the curvature of the non-linear connection and is related to $\Phi$ by the following identity (always with the same restriction to $\pi$-vertical vector fields along $\pi_{1}^{0}$ as explained in the Introduction):

$$
3 R\left(X_{i}, X_{j}\right)=\mathrm{D}_{X_{i}}^{V} \Phi\left(X_{j}\right)-\mathrm{D}_{X_{j}}^{V} \Phi\left(X_{i}\right) .
$$

Making use of the defining relations (2.1) of the structure functions, it is clear that the equality (3.23) will give rise to various relations between these functions, possibly involving the eigenvalues $\lambda_{i}$ of $\Phi$. We shall work them out, first assuming only that $\Phi\left(X_{i}\right)=\lambda_{i} X_{i}$ and $\nabla X_{i}=0$. Taking all possible combinations for $i$ and $j$ in (3.23) and equating each time the coefficients of the two independent terms, one obtains eight equalities which can be represented as follows (there is no summation over repeated indices here, and $i \neq j$ ):

$$
\begin{aligned}
\nabla^{2} \tau_{j j}^{i}+\lambda_{i} \tau_{j j}^{i} & =0, \\
\nabla^{2} \tau_{i i}^{i}+\lambda_{i} \tau_{i i}^{i} & =\mathrm{D}_{X_{i}}^{V} \lambda_{i}, \\
\nabla^{2} \tau_{i j}^{i}+\lambda_{i} \tau_{i j}^{i}+\frac{1}{3}\left(\lambda_{i}-\lambda_{j}\right) \tau_{i j}^{i} & =\frac{2}{3} \mathrm{D}_{X_{j}}^{V} \lambda_{i}, \\
\nabla^{2} \tau_{j i}^{i}+\lambda_{j} \tau_{j i}^{i}-\frac{2}{3}\left(\lambda_{j}-\lambda_{i}\right) \tau_{i j}^{i} & =\frac{1}{3} \mathrm{D}_{X_{j}}^{V} \lambda_{i} .
\end{aligned}
$$

Returning to Case IIa2, we can show that the vector field $Z$ introduced in (3.19) is actually a symmetry of $\Gamma$. Indeed, recalling that

$$
\left[\Gamma, X^{V}\right]=-X^{H}+(\nabla X)^{V}, \quad\left[\Gamma, X^{H}\right]=(\nabla X)^{H}+\Phi(X)^{V},
$$

a direct calculation gives:

$$
[\Gamma, Z]=\left(\Gamma^{2}\left(\tau_{22}^{1}\right)+\lambda_{1} \tau_{22}^{1}\right) X_{1}^{V}=0,
$$

in view of (3.25). Since we know from the theory that compatibility between (3.21) and $\Gamma\left(\varrho_{1}\right)=0$ should be satisfied automatically, this implies that the function $b$ 
defined in (3.20) will be a first integral. It is instructive to double check this: it follows from (3.27) -2 (3.28) that

$$
\nabla^{2}\left(\tau_{i j}^{i}-2 \tau_{j i}^{i}\right)+\lambda_{j}\left(\tau_{i j}^{i}-2 \tau_{j i}^{i}\right)=0 \quad(i \neq j) .
$$

The computation of $\nabla b$, making use of (3.25) again, then simplifies to

$$
\nabla b=-\tau_{22}^{1} \mathrm{D}_{X_{1}}^{V} \lambda_{1}-\nabla \mathrm{D}_{X_{1}}^{V} \nabla \tau_{22}^{1}-\nabla \mathrm{D}_{X_{1}}^{H} \tau_{22}^{1}
$$

Using the commutators (1.11) and (3.23) (the last two terms of which are absent when acting on functions), the right-hand side eventually becomes zero.

The next stage in the integrability analysis of the system (3.2113.22) for $\varrho_{1}$ involves looking at the commutators $\left[X_{2}^{V}, Z\right]$ and $\left[X_{2}^{H}, Z\right]$. For this we need the following bracket relations for horizontal and vertical lifts (the middle one is the same as (A.1) when restricted to functions):

$$
\begin{aligned}
& {\left[X_{i}^{V}, X_{j}^{V}\right]=\left(\mathrm{D}_{X_{i}}^{V} X_{j}-\mathrm{D}_{X_{j}}^{V} X_{i}\right)^{V},} \\
& {\left[X_{i}^{H}, X_{j}^{V}\right]=\left(\mathrm{D}_{X_{i}}^{H} X_{j}\right)^{V}-\left(\mathrm{D}_{X_{j}}^{V} X_{i}\right)^{H}} \\
& {\left[X_{i}^{H}, X_{j}^{H}\right]=\left(\mathrm{D}_{X_{i}}^{H} X_{j}-\mathrm{D}_{X_{j}}^{H} X_{i}\right)^{H}+R\left(X_{i}, X_{j}\right)^{V} .}
\end{aligned}
$$

We shall not give the details of the two new integrability conditions which emerge, but merely explain what can happen by looking at their structure. The point to observe is that the two commutators under investigation can easily be expressed as a linear combination of the vector fields $Z, X_{2}^{H}, X_{2}^{V}$ and $X_{1}^{V}$. Since we have substitutions to make for the first three of these from (3.2113.22), the result is two equations of the same type, say

$$
\begin{aligned}
& A_{1} X_{1}^{V}\left(\varrho_{1}\right)+B_{1}=0 \\
& A_{2} X_{1}^{V}\left(\varrho_{1}\right)+B_{2}=0
\end{aligned}
$$

where the $A_{i}$ and the $B_{i}$ are known functions of the $\tau_{i j}^{k}$ and $\lambda_{i}$. This is in full agreement with the results of Douglas as summarized above. Douglas would say: if, for example, $A_{1}=0$ but $B_{1} \neq 0$, then $\Gamma$ is not variational; likewise for $A_{2}$ and $B_{2}$. Since we are doing these calculations in a more suitable frame than the coordinate vector fields, we can actually do a bit better here and find a certain relationship between (3.35) and (3.36). Let us take one step back and look at the representation of the two equations before the substitutions from (3.2113.22) are done; we then write them as

$$
\begin{aligned}
& {\left[X_{2}^{V}, Z\right]\left(\varrho_{1}\right)=X_{2}^{V}(b)-Z\left(2 \tau_{21}^{1}-\tau_{12}^{1}\right),} \\
& {\left[X_{2}^{H}, Z\right]\left(\varrho_{1}\right)=X_{2}^{H}(b)+Z\left(\Gamma\left(2 \tau_{21}^{1}-\tau_{12}^{1}\right)\right) .}
\end{aligned}
$$

Regardless of the internal consistency of the new conditions (3.35) and (3.36), one normally has to start the integrability procedures again, and the first step would be to check compatibility with the equation $\Gamma\left(\varrho_{1}\right)=0$. But from the Jacobi identity, using the facts that $\nabla X_{2}=0$ and $[Z, \Gamma]=0$, we have

$$
\left.\left[X_{2}^{H}, Z\right]=-\left[\left[\Gamma, X_{2}^{V}\right], Z\right]=\left[\left[X_{2}^{V}, Z\right], \Gamma\right]\right] .
$$

Hence,

$$
\left[X_{2}^{H}, Z\right]\left(\varrho_{1}\right)=\mathcal{L}_{\Gamma}\left(\left[X_{2}^{V}, Z\right]\left(\varrho_{1}\right)\right)
$$


and since $b$ is a first integral and $Z$ commutes with $\Gamma$, we likewise have for the right-hand side of (3.38)

$$
X_{2}^{H}(b)+Z\left(\Gamma\left(2 \tau_{21}^{1}-\tau_{12}^{1}\right)\right)=\mathcal{L}_{\Gamma}\left(X_{2}^{V}(b)-Z\left(2 \tau_{21}^{1}-\tau_{12}^{1}\right)\right) .
$$

It follows that compatibility between (3.37) and $\Gamma\left(\varrho_{1}\right)=0$ reproduces (3.38). One can verify in the same way that compatibility between (3.38) and $\Gamma\left(\varrho_{1}\right)=0$ is (not surprisingly) identically satisfied. Going back to the representation (3.35),(3.36) of the new equations, the conclusion is that also (3.36) is just the Lie derivative with respect to $\Gamma$ of (3.35). But the latter gives

$$
\begin{aligned}
& \Gamma\left(A_{1}\right) X_{1}^{V}\left(\varrho_{1}\right)-A_{1} X_{1}^{H}\left(\varrho_{1}\right)+\Gamma\left(B_{1}\right) \\
& \quad=\Gamma\left(A_{1}\right) X_{1}^{V}\left(\varrho_{1}\right)-\left(\tau_{22}^{1}\right)^{-1} A_{1}\left(Z-\Gamma\left(\tau_{22}^{1}\right) X_{1}^{V}\right)\left(\varrho_{1}\right)+\Gamma\left(B_{1}\right) .
\end{aligned}
$$

We conclude therefore that

$$
A_{2} \propto \Gamma\left(\tau_{22}^{1} A_{1}\right), \quad B_{2} \propto \tau_{22}^{1} \Gamma\left(B_{1}\right)-A_{1} b .
$$

This means that one of the situations discussed by Douglas cannot occur, because if the first condition (3.35) is void, the second one 3.36 is void as well $\left(A_{1}=\right.$ $\left.B_{1}=0 \Rightarrow A_{2}=B_{2}=0\right)$. The final list of possibilities in Case IIa2 thus becomes somewhat simpler and reads as follows: (i) if $A_{1}=0$ and $B_{1} \neq 0$, then there is no solution, i.e. the system is not variational; (ii) if $A_{1}=B_{1}=0$, then the equations $3.21+3.22$ for $\varrho_{1}$ are formally integrable, and so are subsequently the three equations for $\rho_{2}$, and for what it is worth, the freedom in the solution is one function of one variable and one function of two variables; (iii) if $A_{1} \neq 0$, then also $A_{2} \neq 0$, and to have a solution, we must first of all have that the equations (3.35) and (3.36) are linearly dependent, i.e.,

$$
\left(A_{1} \tau_{22}^{1}\right) \Gamma\left(B_{1}\right)-\Gamma\left(A_{1} \tau_{22}^{1}\right) B_{1}=A_{1}^{2} b
$$

with (3.40) satisfied, we have equations for all first-order derivatives of $\varrho_{1}$, and their integrability is a standard test which amounts to a certain 1-form being closed; if that happens then the freedom in the solution is one function of two variables (coming from the equations for $\rho_{2}$ ). All of this is in agreement with the results of Douglas, and actually makes them more precise on a number of points. In fact, it would not be too hard to go further and compute explicitly the obstructions for the closure of the 1-form under consideration; they come from the compatibility between (3.35) and each of the equations (3.21,3.22) with the exception of $\Gamma\left(\varrho_{1}\right)=0$. But perhaps it is not very fruitful to do this in such a general discussion; it can easily be done for each example in this category. This concludes our treatment of Case IIa2.

What about Case IIa3, the "non-separated case", i.e., the case where both $\tau_{22}^{1} \neq$ 0 and $\tau_{11}^{2} \neq 0$ ? Obviously, this discussion is bound to be even more complicated, so we will not carry it out here. It is instructive to see, however, how our treatment of the previous case directly gives some clues about addressing the present issue. Going back to the final $\mathcal{A}$-condition (3.11) we obtained before we started using simplifications coming from assumptions on $H_{\Phi}$, it is obvious that there are two parts in it which can be mapped into each other by interchanging 1 and 2 , and that the whole discussion in Case IIa2 was about one of these parts. So, we should write (3.11) in the form

$$
Z_{1}\left(\rho_{1}\right)-b_{1} \rho_{1}=Z_{2}\left(\rho_{2}\right)-b_{2} \rho_{2},
$$

where $Z_{1}$ and $b_{1}$ are the $Z$ and $b$ defined respectively in (3.19) and (3.20), and $Z_{2}$ and $b_{2}$ are their analogues with 1 and 2 interchanged. The direct verification we 
did before, proving that $Z$ is a symmetry of $\Gamma$ and $b$ is a first integral, remains unaltered and applies to both $Z_{i}$ and $b_{i}$ here. As a kind of separation of variables technique, it looks plausible next to introduce an auxiliary unknown, $\tau$ say, which represents both sides of (3.41). Not surprisingly, this is precisely what Douglas did to continue the integrability analysis of this case. But we have much more accurate information about the quantities involved now, so we can undoubtedly sharpen Douglas's results in this case as well. For example, it follows from the properties of $Z_{i}$ and $b_{i}$ that we will have $\Gamma(\tau)=0$, which is easier to handle than the non-zero expression Douglas had (in fact, he mentions having to compute two expressions for $\Gamma(\tau)$, which happen to coincide). We leave it to the reader to complete this discussion.

We shall now depart from Case IIa by assuming that $[\nabla \Phi, \Phi] \neq 0$. The algebraic freedom in $g$ will then further be restricted by the condition (1.12) for $k=1$. In fact, in order to have a solution for the inverse problem, all further conditions in the hierarchy (1.12) must be satisfied identically, so we have to assume that

$$
\nabla^{2} \Phi=a \nabla \Phi+b \Phi+c I, \quad a, b, c \in C^{\infty}\left(J^{1} \pi\right) .
$$

This is Case III of Douglas, which is further subdivided into two subcases, but only in the first of these, Case IIIa, is there a chance for finding a non-degenerate solution for $g$. It was shown in 4 that $\Phi$ is then necessarily diagonalizable again. So, we assume we are in this situation now. This time, all we can achieve by rescaling the eigenvectors $X_{i}$ is to have $\nu_{i}^{i}=0$, i.e.

$$
\nabla X_{1}=\nu_{1}^{2} X_{2}, \quad \nabla X_{2}=\nu_{2}^{1} X_{1}
$$

With $g\left(X_{1}, X_{2}\right)=0$ from the condition (1.6) as before, 1.12) for $k=1$ is equivalent to $g\left(\nabla X_{1}, X_{2}\right)+g\left(X_{1}, \nabla X_{2}\right)=0$, which implies $\nu_{2}^{1} \rho_{1}+\nu_{1}^{2} \rho_{2}=0$. It further follows that $\alpha=\nu_{2}^{1} / \nu_{1}^{2}$ is a first integral which is known in principle (see [4]). Hence, any algebraically admissible $g$ is of the form

$$
g=\rho_{1}\left(\theta^{1} \otimes \theta^{1}-\alpha \theta^{2} \otimes \theta^{2}\right), \quad \text { with } \quad \Gamma(\alpha)=0 .
$$

Turning to the differential conditions for the only unknown function $\rho_{1}, \nabla g=0$ requires that $\Gamma\left(\rho_{1}\right)=0$, while the closure conditions 3.23 .5 become

$$
\begin{aligned}
\mathrm{D}_{X_{2}}^{V} \rho_{1} & =\left(2 \tau_{21}^{1}-\tau_{12}^{1}+\alpha \tau_{11}^{2}\right) \rho_{1}, \\
\alpha \mathrm{D}_{X_{1}}^{V} \rho_{1} & =\left(\alpha\left(2 \tau_{12}^{2}-\tau_{21}^{2}\right)+\tau_{22}^{1}-\mathrm{D}_{X_{1}}^{V} \alpha\right) \rho_{1}, \\
\mathrm{D}_{X_{2}}^{H} \rho_{1} & =\left(2 \mu_{21}^{1}-\mu_{12}^{1}+\alpha \mu_{11}^{2}\right) \rho_{1}, \\
\alpha \mathrm{D}_{X_{1}}^{H} \rho_{1} & =\left(\alpha\left(2 \mu_{12}^{2}-\mu_{21}^{2}\right)+\mu_{22}^{1}-\mathrm{D}_{X_{1}}^{H} \alpha\right) \rho_{1} .
\end{aligned}
$$

This is a typical situation where the linear dependence between $\rho_{1}$ and $\rho_{2}$ has made the ordering become improper: we have two vertical derivatives of $\rho_{1}$ in the list of principal derivatives (after division by $\alpha$ in (3.46) ) and the corresponding right-hand sides are not derivatives of $g$ with respect to the same vector argument. As a result, we can no longer trust that the single $\mathcal{A}$-condition (3.11) would constitute the only second-order passivity condition. In fact, it is manifestly clear that there will, for example, be a $\mathrm{D}^{V} \mathrm{D}^{V}$-compatibility condition coming from (3.45) and (3.46). Since we have equations for all derivatives of the single unknown $\rho_{1}$, however, we can say (with Douglas) that integrability now is a matter of a certain 1-form being closed. The verification of this is a standard process so, rather than working out all mixed 
derivative conditions coming from (3.45 $[3.48)$ in all generality, we can safely leave that to each explicit example falling in this category.

\section{The Cases Where $\Phi$ is not Diagonalizable}

There is one main category in Douglas's classification which we have not touched upon yet, namely Case IIb. So assume again that $[\nabla \Phi, \Phi]=0$, but that this time $\Phi$ is not diagonalizable. We choose a local frame of vector fields along $\pi_{1}^{0}$ such that

$$
\begin{aligned}
& \Phi\left(X_{1}\right)=\lambda X_{1}, \\
& \Phi\left(X_{2}\right)=a X_{1}+\lambda X_{2} \quad a \neq 0 .
\end{aligned}
$$

The algebraic condition (1.6) then requires that $g\left(X_{1}, X_{1}\right)=0$. The next one in the hierarchy would read $g\left(\nabla X_{1}, X_{1}\right)=0$, but since $\nabla \Phi$ can be expressed as a linear combination of $\Phi$ and the identity, this condition has to be void, meaning that $\nabla X_{1}$ will be proportional to $X_{1}$ and thus can be rescaled to be zero. So, we may assume here that the $X_{i}$ are chosen in such a way that

$$
\nabla X_{1}=0, \quad \nabla X_{2}=\nu_{2}^{1} X_{1},
$$

and $g$ is bound to be of the form (with respect to dual 1-forms)

$$
g=\rho_{2} \theta^{2} \otimes \theta^{2}+\rho\left(\theta^{1} \otimes \theta^{2}+\theta^{2} \otimes \theta^{1}\right) .
$$

The closure conditions (2.7:2.10) with $\rho_{1}=0$ can be written in the form

$$
\begin{aligned}
\mathrm{D}_{X_{1}}^{V} \rho & =\rho_{A}\left(v_{21}^{A}-v_{13}^{A}\right), \\
\mathrm{D}_{X_{1}}^{V} \rho_{2} & =\mathrm{D}_{X_{2}}^{V} \rho+\rho_{A}\left(v_{23}^{A}-v_{12}^{A}\right), \\
\mathrm{D}_{X_{1}}^{H} \rho & =\rho_{A}\left(\kappa_{21}^{A}-\kappa_{13}^{A}\right), \\
\mathrm{D}_{X_{1}}^{H} \rho_{2} & =\mathrm{D}_{X_{2}}^{H} \rho+\rho_{A}\left(\kappa_{23}^{A}-\kappa_{12}^{A}\right) .
\end{aligned}
$$

Written in this form, the ordering is proper, so the rule concerning the $\mathcal{A}$-conditions says that there is again only one condition to look at (this time different from (2.12) however), namely

$$
\left(\mathcal{A}\left(X_{1}, X_{2}\right) g\right)\left(X_{1}, X_{1}\right)=0 .
$$

We sketch in a few steps how the computation goes. A good starting point is the general expression (2.11), which we apply to the pair $\left(X_{1}, X_{1}\right)$, knowing further that $\rho_{1}=0$. Clearly, there are no second-order terms; we further make substitutions for all first-order $X_{1}$-derivatives from the above closure conditions. Expressing everything in terms of $\tau_{i j}^{k}$ and $\mu_{i j}^{k}$ by using (2.6), the part which does not involve the $\theta$-tensor eventually becomes

$$
\begin{gathered}
\left(A\left(X_{1}, X_{2}\right) g\right)\left(X_{1}, X_{1}\right)=2 \mu_{11}^{2} \mathrm{D}_{X_{2}}^{V} \rho-2 \mu_{11}^{2} \mathrm{D}_{X_{2}}^{H} \rho+\rho\left[-2 \mathrm{D}_{X_{1}}^{V} \mu_{21}^{2}+2 \mathrm{D}_{X_{2}}^{V} \mu_{11}^{2}\right. \\
\left.-2 \mu_{21}^{2}\left(\tau_{21}^{2}-\tau_{11}^{1}\right)+2 \mu_{11}^{2}\left(\tau_{12}^{1}-3 \tau_{21}^{1}\right)+2 \tau_{11}^{2}\left(\mu_{21}^{1}+\mu_{22}^{2}\right)\right] .
\end{gathered}
$$

To this we must add the terms

$$
\begin{gathered}
g\left(\theta\left(X_{1}, X_{1}\right) X_{1}, X_{2}\right)-g\left(X_{1}, \theta\left(X_{1}, X_{1}\right), X_{1}\right)=\rho_{2}\left\langle\theta\left(X_{1}, X_{1}\right) X_{1}, \theta^{2}\right\rangle \\
+\rho\left[\left\langle\theta\left(X_{1}, X_{1}\right) X_{1}, \theta^{1}\right\rangle-\left\langle\theta\left(X_{1}, X_{1}\right) X_{2}, \theta^{2}\right\rangle\right] .
\end{gathered}
$$

As before, we need to make a choice concerning the way in which we compute the $\theta$-tensors. The coefficient of $\rho_{2}$ which emerges here starts with $X_{1}^{V}$ and $X_{1}^{H}$ derivatives of structure functions; the same will be true for the term in $\rho$ coming 
from $\left\langle\theta\left(X_{1}, X_{1}\right) X_{1}, \theta^{1}\right\rangle$, where there is no freedom of choice; in the last term, we have a choice for three different looking (but equivalent) expressions; for the sake of the overall symmetry in the formula, an objective then can be to eliminate the term $2 \mathrm{D}_{X_{2}}^{V} \mu_{11}^{2}$ from the coefficient of $\rho$ without introducing other than $X_{1}$-derivatives; this is achieved if we compute the last term according to the property

$$
\left\langle\theta\left(X_{1}, X_{1}\right) X_{2}, \theta^{2}\right\rangle=2\left\langle\theta\left(X_{2}, X_{1}\right) X_{1}, \theta^{2}\right\rangle-\left\langle\theta\left(X_{1}, X_{1}\right) X_{2}, \theta^{2}\right\rangle .
$$

Putting everything together then, we reach the following general expression for the condition (4.9) (which will be valid as long as it makes sense to write the closure conditions with principal derivatives as in (4.5.4.8)):

$$
\begin{aligned}
0= & 2 \mu_{11}^{2} \mathrm{D}_{X_{2}}^{V} \rho-2 \tau_{11}^{2} \mathrm{D}_{X_{2}}^{H} \rho+\rho\left[\mathrm{D}_{X_{1}}^{V}\left(\mu_{11}^{1}-2 \mu_{21}^{2}+\mu_{12}^{2}\right)\right. \\
& -\mathrm{D}_{X_{1}}^{H}\left(\tau_{11}^{1}-2 \tau_{21}^{2}+\tau_{12}^{2}\right)+\tau_{11}^{1}\left(2 \mu_{21}^{2}-\mu_{12}^{2}\right)-\mu_{11}^{1}\left(2 \tau_{21}^{2}-\tau_{12}^{2}\right) \\
& \left.+\mu_{11}^{2}\left(2 \tau_{12}^{1}-\tau_{21}^{1}-\tau_{22}^{2}\right)-\tau_{11}^{2}\left(2 \mu_{12}^{1}-\mu_{21}^{1}-\mu_{22}^{2}\right)\right] \\
& +\rho_{2}\left[\mathrm{D}_{X_{1}}^{V} \mu_{11}^{2}-\mathrm{D}_{X_{1}}^{H} \tau_{11}^{2}+\tau_{11}^{2}\left(2 \mu_{11}^{1}-\mu_{12}^{2}-\mu_{21}^{2}\right)-\mu_{11}^{2}\left(2 \tau_{11}^{1}-\tau_{21}^{2}-\tau_{12}^{2}\right)\right] .
\end{aligned}
$$

The relationship between the $\mu_{i j}^{k}$ and $\tau_{i j}^{k}$ follows from (1.11) as before. To be concrete, computing the identity $\left[\nabla, \mathrm{D}_{X_{i}}^{V}\right] X_{j}=\mathrm{D}_{\nabla X_{i}}^{V} X_{j}-\mathrm{D}_{X_{i}}^{H} X_{j}$, taking account of the simplifications (4.3), one easily finds that

$$
\mu_{i j}^{k}=-\nabla \tau_{i j}^{k}-\tau_{i j}^{2} \nu_{2}^{1} \delta_{1}^{k}+\delta_{j}^{2}\left(\delta_{1}^{k} \mathrm{D}_{X_{i}}^{V} \nu_{2}^{1}+\nu_{2}^{1} \tau_{i 1}^{k}\right)+\delta_{i}^{2} \nu_{2}^{1} \tau_{1 j}^{k},
$$

which means for example that, in particular,

$$
\mu_{11}^{2}=-\nabla \tau_{11}^{2} .
$$

We will not, at this level of generality, list the eight further relations (involving also the entries $\lambda$ and $\alpha$ of $\Phi$ ) which follow from the identity (3.23). The simplest one of them reads

$$
\nabla \mu_{11}^{2}=\lambda \tau_{11}^{2} .
$$

It follows from (4.14) and (4.15) as before that the vector field $\tau_{11}^{2} X_{2}^{H}-\mu_{11}^{2} X_{2}^{V}$, which determines the first-order part in the integrability condition (4.12), is a symmetry of $\Gamma$. This extra information will undoubtedly be useful again, if one were to analyse completely Case IIb2 of Douglas, which is the case where $\tau_{11}^{2} \neq 0$, so that the $\mathcal{A}$-condition (4.12) does not further degenerate into an algebraic condition. In Douglas's analysis, however, this case gives rise to numerous complicated subcases; we shall therefore not push this discussion through until the end, even though there is little doubt that we should obtain more accurate conclusions for this situation also.

Let us then concentrate on the more manageable Case IIb1, which is the case where

$$
\tau_{11}^{2}=0
$$

The reason Douglas singled out this case is again that it corresponds to a decoupling of the closure conditions: it is easy to see that with $\tau_{11}^{2}=0$, the equations (4.5) and (4.7) for $\rho$ decouple from the equations for $\rho_{2}$. The geometrical interpretation of (4.16) is $H_{\Phi}=0$ again. To see this, let us write $\Phi$, as determined by (4.14.2), as

$$
\Phi=\lambda I+a N,
$$


with $N X_{1}=0, N X_{2}=X_{1}$, and hence $N^{2}=0$. From the defining relations (3.15) 3.16$)$, one then obtains

$$
H_{\Phi}\left(X_{i}, X_{j}\right)=a^{2}\left(\mathrm{D}^{V} \Phi\left(N X_{i}, N X_{j}\right)-N\left(\mathrm{D}^{V} \Phi\left(X_{i}, N X_{j}\right)+\mathrm{D}^{V} \Phi\left(N X_{i}, X_{j}\right)\right),\right.
$$

with

$$
\begin{aligned}
& \mathrm{D}^{V} \Phi\left(X_{i}, X_{j}\right) \equiv \mathrm{D}_{X_{i}}^{V} \Phi\left(X_{j}\right) \\
& \quad=\left(\mathrm{D}_{X_{i}}^{V} \lambda\right) X_{j}+\left(\mathrm{D}_{X_{i}}^{V} a\right) N X_{j}+a\left(\mathrm{D}_{X_{i}}^{V}\left(N X_{j}\right)-N\left(\mathrm{D}_{X_{i}}^{V} X_{j}\right),\right.
\end{aligned}
$$

which ultimately results in the simple expression

$$
H_{\Phi}\left(X_{i}, X_{j}\right)=-2 a^{3} N\left(\mathrm{D}_{N X_{i}}^{V}\left(N X_{j}\right)\right) .
$$

The only component of this which is not identically zero is the one for $i=j=2$, and $N\left(\mathrm{D}_{X_{1}}^{V} X_{1}\right)=0$ is clearly equivalent to (4.16).

In view of (4.14), we also have $\mu_{11}^{2}=0$ here, so the $\mathcal{A}$-condition (4.12) drastically reduces to an algebraic requirement of the form

$$
b \rho=0,
$$

with

$$
\begin{aligned}
b= & \mathrm{D}_{X_{1}}^{V}\left(\mu_{11}^{1}-2 \mu_{21}^{2}+\mu_{12}^{2}\right)-\mathrm{D}_{X_{1}}^{H}\left(\tau_{11}^{1}-2 \tau_{21}^{2}+\tau_{12}^{2}\right) \\
& +\tau_{11}^{1}\left(2 \mu_{21}^{2}-\mu_{12}^{2}\right)-\mu_{11}^{1}\left(2 \tau_{21}^{2}-\tau_{12}^{2}\right) .
\end{aligned}
$$

Can anything more be said about the known function $b$ ? Observe first that with $g$ of the form (4.4) and the $X_{i}$ satisfying (4.3), it follows that the equation $\nabla g=0$ requires

$$
\nabla \rho=0, \quad \nabla \rho_{2}=2 \nu_{2}^{1} \rho .
$$

In view of our general claim that $\mathcal{A}$-conditions always are compatible with the equation $\nabla g=0$, we should have that $b$ is a first integral. Let us once more indicate how this can be verified explicitly. With $\tau_{11}^{2}=0$, there are some simplifications in the relations (4.13) which result in the following property holding true:

$$
\mu_{11}^{1}-2 \mu_{21}^{2}+\mu_{12}^{2}=-\nabla\left(\tau_{11}^{1}-2 \tau_{21}^{2}+\tau_{12}^{2}\right) .
$$

Also the eight relations following from the identity (3.23) become somewhat simpler. Among them we find that

$$
\begin{aligned}
\mathrm{D}_{X_{1}}^{V} \lambda & =-\nabla \mu_{11}^{1}+\lambda \tau_{11}^{1}=\nabla^{2} \tau_{11}^{1}+\lambda \tau_{11}^{1}, \\
\frac{1}{3} \mathrm{D}_{X_{1}}^{V} \lambda & =-\nabla \mu_{12}^{2}+\lambda \tau_{12}^{2}=\nabla^{2} \tau_{12}^{2}+\lambda \tau_{12}^{2}, \\
\frac{2}{3} \mathrm{D}_{X_{1}}^{V} \lambda & =-\nabla \mu_{21}^{2}+\lambda \tau_{21}^{2}=\nabla^{2} \tau_{21}^{2}+\lambda \tau_{21}^{2},
\end{aligned}
$$

from which it follows that

$$
\nabla^{2}\left(\tau_{11}^{1}-2 \tau_{21}^{2}+\tau_{12}^{2}\right)+\lambda\left(\tau_{11}^{1}-2 \tau_{21}^{2}+\tau_{12}^{2}\right)=0 .
$$

With the properties (4.22 4.23) it is easy to check that the function $b$ of (4.20) is indeed a first integral.

In any case, the conclusions which we find for Case IIb1 are very simple (and in agreement with Douglas): (i) if $b \neq 0$, then the system is not variational (choosing $\rho=0$ would violate the regularity condition for $g$ ); (ii) if $b \equiv 0$, then the system is variational and the freedom in the solution for $g$ is two functions of two variables 
(for each of the unknowns $\rho$ and $\rho_{2}$, three of their five first-order partial derivatives are determined by (4.514.8) and (4.21)).

This discussion of Case IIb1 gives us an opportunity to illustrate explicitly that the possible occurrence of higher-order passivity conditions in Riquier's approach to formal integrability is fully consistent, even if we select an ordering which is not proper. Going back to the start of the section, suppose we choose to write the closure conditions (4.54.8) in the following way (which in some respects may in fact seem to be more logical):

$$
\begin{aligned}
& \mathrm{D}_{X_{1}}^{V} \rho=\rho_{A}\left(v_{21}^{A}-v_{13}^{A}\right), \\
& \mathrm{D}_{X_{2}}^{V} \rho=\mathrm{D}_{X_{1}}^{V} \rho_{2}-\rho_{A}\left(v_{23}^{A}-v_{12}^{A}\right), \\
& \mathrm{D}_{X_{1}}^{H} \rho=\rho_{A}\left(\kappa_{21}^{A}-\kappa_{13}^{A}\right), \\
& \mathrm{D}_{X_{2}}^{H} \rho=\mathrm{D}_{X_{1}}^{H} \rho_{2}-\rho_{A}\left(\kappa_{23}^{A}-\kappa_{12}^{A}\right) .
\end{aligned}
$$

With reference to the original vertical closure conditions (1.4), the left-hand sides of (4.24) and 4.25) come from $\mathrm{D}_{X_{1}}^{V} g\left(X_{1}, X_{2}\right)$ and $\mathrm{D}_{X_{2}}^{V} g\left(X_{1}, X_{2}\right)$, respectively. The right-hand sides correspondingly originate from $\mathrm{D}_{X_{2}}^{V} g\left(X_{1}, X_{1}\right)$ and $\mathrm{D}_{X_{1}}^{V} g\left(X_{2}, X_{2}\right)$, which are components of derivatives of $g$ with respect to different vector arguments. Hence, the ordering is not proper, and there may be more second-order passivity conditions than those coming from $\mathcal{A}$-conditions. Without going into details, a sketch of what one will obtain in the simplified situation $H_{\Phi}=0$ goes as follows. The computation of the bracket $\left[X_{1}^{V}, X_{1}^{H}\right](\rho)$ produces an algebraic condition which is (4.19) again. All the other mixed derivatives give rise to second-order conditions on $\rho_{2}$, but the ones coming from $\left[X_{1}^{H}, X_{2}^{V}\right](\rho)$ and $\left[X_{1}^{V}, X_{2}^{H}\right](\rho)$ coincide. No further passivity conditions can be obtained. Although the present set of conditions looks very different from what we had before, the overall conclusion is the same: provided $b=0$, the system is variational. To count the freedom in the solution: we have equations for all first-order derivatives of $\rho$, leaving no arbitrariness there; for $\rho_{2}$, on the other hand, we have the equation for $\nabla \rho_{2}$ coming from $\nabla g=0$, plus four second-order conditions; it can be verified by drawing up the appropriate table of parametric derivatives and multipliers (see 13 for details) that the freedom in the solution is again two functions of two variables.

\section{EXAMPLES}

As a first example, we consider the system

$$
\begin{aligned}
& \ddot{x}_{1}=\frac{1}{x_{1}}\left(1+\dot{x}_{1}^{2}+\dot{x}_{2}^{2}\right) \quad\left(=f_{1}\right), \\
& \ddot{x}_{2}=0
\end{aligned}
$$

which is taken from [5] and should fall into Case IIa2. The Jacobi endomorphism is given by

$$
\left(\Phi_{j}^{i}\right)=\frac{2}{x_{1}^{2}}\left(\begin{array}{cc}
1+\dot{x}_{2}^{2} & -\dot{x}_{1} \dot{x}_{2} \\
0 & 0
\end{array}\right)
$$

and has eigenvalues $\lambda_{1}=2 x_{1}^{-2}\left(1+\dot{x}_{2}^{2}\right), \lambda_{2}=0$. One easily verifies that $[\nabla \Phi, \Phi]=0$, so we are certainly in Case IIa. Eigenvectors of $\Phi$ which are $\nabla$-invariant are given 
by

$$
X_{1}=x_{1} \frac{\partial}{\partial x_{1}}, \quad X_{2}=\dot{x}_{1} \dot{x}_{2} \frac{\partial}{\partial x_{1}}+\left(1+\dot{x}_{2}^{2}\right) \frac{\partial}{\partial x_{2}} .
$$

We then have

$$
\mathrm{D}_{X_{i}}^{V} X_{1}=0, \quad \mathrm{D}_{X_{1}}^{V} X_{2}=\dot{x}_{2} X_{1}, \quad \mathrm{D}_{X_{2}}^{V} X_{2}=\frac{\dot{x}_{1}}{x_{1}} X_{1}+2 \dot{x}_{2} X_{2},
$$

from which it follows in particular that $\tau_{11}^{2}=0$, but $\tau_{22}^{1}=\dot{x}_{1} / x_{1} \neq 0$, so that we are in the "semi-separated" case indeed. The function $b$ as defined by (3.20) turns out to be zero here. Slightly more tedious is the calculation which leads to the conclusion that also $A_{1}=B_{1}=0$ (cf. (3.35)). Hence, no further integrability conditions can be obtained beyond the $\mathcal{A}$-condition (3.18), which simply reads $Z\left(\rho_{1}\right)=0$. The other equations to be satisfied by $\rho_{1}$, namely (3.22), become

$$
\Gamma\left(\rho_{1}\right)=0, \quad X_{2}^{V}\left(\rho_{1}\right)=-\dot{x}_{2} \rho_{1}, \quad X_{2}^{H}\left(\rho_{1}\right)=0 .
$$

Note that

$$
Z=\dot{x}_{1} \frac{\partial}{\partial x_{1}}+f_{1} \frac{\partial}{\partial \dot{x}_{1}}=\Gamma-\dot{x}_{2} \frac{\partial}{\partial x_{2}}-\frac{\partial}{\partial t},
$$

and that

$$
X_{2}^{H}=\dot{x}_{2} Z+\left(1+\dot{x}_{2}^{2}\right) \frac{\partial}{\partial x_{2}} .
$$

It thus readily follows that $\rho_{1}$ cannot depend on $x_{2}$, nor on $t$, and further must be a first integral, satisfying $X_{2}^{V}\left(\rho_{1}\right)=-\dot{x}_{2} \rho_{1}$. This leaves the freedom of an arbitrary function of the variable

$$
\frac{1}{x_{1}} \sqrt{\frac{1+\dot{x}_{1}^{2}+\dot{x}_{2}^{2}}{1+\dot{x}_{2}^{2}}}
$$

in the solution for $\rho_{1}$. The remaining conditions (3.3) and (3.5), together with the requirement $\Gamma\left(\rho_{2}\right)=0$, subsequently leave a freedom of a function of two variables in the solution for $\rho_{2}$. Observe finally that the vector field $Z$ is indeed a symmetry of $\Gamma$.

Another example taken from Douglas is the system

$$
\begin{aligned}
& \ddot{x}_{1}=x_{2}^{2}, \\
& \ddot{x}_{2}=x_{1}^{2} .
\end{aligned}
$$

Again, $\Phi$ is diagonalizable: the eigenvalues are $\lambda_{1}=-2 \sqrt{x_{1} x_{2}}, \lambda_{2}=2 \sqrt{x_{1} x_{2}}$. We have $[\nabla \Phi, \Phi] \neq 0$, but (3.42) is satisfied; thus we are in Case IIIa. Eigenvectors, suitably rescaled to satisfy (3.43), are given by

$$
X_{1}=\left(\frac{x_{2}}{x_{1}}\right)^{1 / 4} \frac{\partial}{\partial x_{1}}+\left(\frac{x_{1}}{x_{2}}\right)^{1 / 4} \frac{\partial}{\partial x_{2}}, \quad X_{2}=-\left(\frac{x_{2}}{x_{1}}\right)^{1 / 4} \frac{\partial}{\partial x_{1}}+\left(\frac{x_{1}}{x_{2}}\right)^{1 / 4} \frac{\partial}{\partial x_{2}} .
$$

Since these are basic vector fields, all $\tau_{i j}^{k}$ vanish. We further have $\nu_{1}^{2}=\nu_{2}^{1}$, so that the first integral $\alpha$ in the representation (3.44) of $g$ is simply the constant 1 . Since the $X_{i}$ are basic and the connection coefficients $\Gamma_{j}^{i}$ are zero, the vector fields $\mathrm{D}_{X_{i}}^{H} X_{j}$ will be basic as well. This in turn implies that the structure functions $\mu_{i j}^{k}$ will be basic. In fact, one can easily verify that the closure conditions (3.45),3.48) become

$$
\mathrm{D}_{X_{i}}^{V} \rho_{1}=0, \quad \mathrm{D}_{X_{i}}^{H} \rho_{1}=0, \quad i=1,2 .
$$


The ordering here is not proper, but it is obvious that this system is integrable and limits the unknown $\rho_{1}$ in $g$ to be a constant $(1$, say). It is perhaps worth mentioning that while $g$ is diagonal in the eigenform basis $\theta^{i}$, we find in the coordinate basis that

$$
g=\frac{1}{2}\left(d x_{1} \otimes d x_{2}+d x_{2} \otimes d x_{1}\right)
$$

leading to the Lagrangian

$$
L=\frac{1}{2} \dot{x}_{1} \dot{x}_{2}+\frac{1}{6}\left(x_{1}^{3}+x_{2}^{3}\right) .
$$

A simple example belonging to Case IIb1 is the following:

$$
\begin{aligned}
& \ddot{x}_{1}=2 f\left(x_{2}, \dot{x}_{2}\right), \\
& \ddot{x}_{2}=0 .
\end{aligned}
$$

$\Phi$ here is of the form (4.17) with respect to the coordinate vector fields, with $\lambda=0$ and $a=-2\left(\partial f / \partial x_{2}\right)+\dot{x}_{2}\left(\partial^{2} f / \partial x_{2} \partial \dot{x}_{2}\right)$. The coordinate vector fields already have the scaling properties (4.3), with $\nu_{2}^{1}=-\partial f / \partial \dot{x}_{2}$. Clearly, all $\tau_{i j}^{k}$ are zero and, from (4.13), all $\mu_{i j}^{k}$ are found to be zero as well, with the exception of $\mu_{22}^{1}$. It then follows from (4.20) that $b=0$, so that the system is variational.

The following example is taken from the monograph [1]:

$$
\begin{aligned}
& \ddot{x}_{1}=-\phi_{x_{1}} \dot{x}_{1}^{2}, \\
& \ddot{x}_{2}=\phi_{x_{2}} \dot{x}_{2}^{2},
\end{aligned}
$$

where $\phi\left(x_{1}, x_{2}\right)$ is arbitrary except for the assumption $\phi_{x_{1} x_{2}} \neq 0$. The Jacobi endomorphism $\Phi$ has a double eigenvalue $\lambda=0$ and is not diagonalizable, but $[\nabla \Phi, \Phi]=0$, so we are in Case IIb to start with. Choosing

$$
X_{1}=\dot{x}_{1} \frac{\partial}{\partial x_{1}}+\dot{x}_{2} \frac{\partial}{\partial x_{2}}, \quad X_{2}=\dot{x}_{1} \frac{\partial}{\partial x_{1}}-\dot{x}_{2} \frac{\partial}{\partial x_{2}},
$$

we have $\Phi\left(X_{1}\right)=0$ and $\Phi\left(X_{2}\right)=-2 \phi_{x_{1} x_{2}} \dot{x}_{1} \dot{x}_{2} X_{1}$ (which identifies the function $a$ in (4.2)). It turns out that both $X_{i}$ are $\nabla$-invariant, so that $\nu_{2}^{1}=0$. We further get the following structure equations:

$$
\mathrm{D}_{X_{1}}^{V} X_{1}=X_{1}, \quad \mathrm{D}_{X_{1}}^{V} X_{2}=X_{2}, \quad \mathrm{D}_{X_{2}}^{V} X_{1}=X_{2}, \quad \mathrm{D}_{X_{2}}^{V} X_{2}=X_{1},
$$

from which we see that all $\tau_{i j}^{k}$ are either zero or one. In particular, we have $\tau_{11}^{2}=0$, so that we are in fact in Case IIb1 again, and the function $b$ defined by (4.20) determines whether the system is variational or not. It follows from (4.13) that all $\mu_{i j}^{k}$ are zero, and thus obviously also $b=0$. The closure conditions (4.5,4.8) explicitly become

$$
\begin{aligned}
& X_{1}^{V}(\rho)=0, \quad X_{1}^{V}\left(\rho_{2}\right)=X_{2}^{V}(\rho)+\rho_{2}, \\
& X_{1}^{H}(\rho)=0, \quad X_{1}^{H}\left(\rho_{2}\right)=X_{2}^{H}(\rho),
\end{aligned}
$$

and both $\rho$ and $\rho_{2}$ have to be first integrals. The freedom in the solution is clearly two functions of two variables. We shall not determine the complete solution explicitly, but observe in passing that $X_{1}^{H}=\Gamma-\partial / \partial t$, from which it follows that $\rho$ cannot depend on $t$, whereas $\rho_{2}$ can be at most linear in $t$. One can verify in detail here that if one selects all derivatives of $\rho$ in the above system as principal derivatives, there will be four second-order passivity conditions to be satisfied by $\rho_{2}$. This has no effect on the conclusion about the solution. 
A minor variation on the above example will bring us back to a Case IIa2 situation. Consider the system

$$
\begin{aligned}
& \ddot{x}_{1}=-\frac{\phi_{x_{1}}}{p} \dot{x}_{1}^{2}, \\
& \ddot{x}_{2}=-\frac{\phi_{x_{2}}}{q} \dot{x}_{2}^{2},
\end{aligned}
$$

with $p, q \in \mathbb{R} \backslash\{0\}$ and $p+q \neq 0$. $\Phi$ this time has distinct eigenvalues, and the corresponding normalized eigenvectors are given by

$$
X_{1}=\dot{x}_{1} \frac{\partial}{\partial x_{1}}+\dot{x}_{2} \frac{\partial}{\partial x_{2}}, \quad X_{2}=q \dot{x}_{1} \frac{\partial}{\partial x_{1}}-p \dot{x}_{2} \frac{\partial}{\partial x_{2}} .
$$

We have

$$
\begin{array}{ll}
\mathrm{D}_{X_{1}}^{V} X_{1}=X_{1}, & \mathrm{D}_{X_{1}}^{V} X_{2}=(p-q) X_{1}+X_{2}, \\
\mathrm{D}_{X_{2}}^{V} X_{1}=X_{2}, & \mathrm{D}_{X_{2}}^{V} X_{2}=p q X_{1}+(q-p) X_{2} .
\end{array}
$$

All $\tau_{i j}^{k}$ are constants again, implying via (3.12) that the $\mu_{i j}^{k}$ are zero. Since $\tau_{11}^{2}=0$ and $\tau_{22}^{1}=p q$, we are indeed in the 'semi-separated case'. The function $b$ from (3.20) is zero, and the symmetry vector field $Z$ is given by

$$
Z=p q X_{1}^{H}=p q\left(\Gamma-\frac{\partial}{\partial t}\right) .
$$

Since $\rho_{1}$ has to be a first integral, it follows that the condition $Z\left(\rho_{1}\right)=0$ essentially means that $\rho_{1}$ does not depend on time. The other equations (3.22) to be satisfied by $\rho_{1}$ read

$$
X_{2}^{V}\left(\rho_{1}\right)=(q-p) \rho_{1}, \quad X_{2}^{H}\left(\rho_{1}\right)=0 .
$$

It is then immediately clear that confronting these conditions with $Z\left(\rho_{1}\right)=0$ does not produce further integrability conditions. In other words, the functions $A_{i}$ and $B_{i}$ in (3.35), (3.36) are bound to be zero, and the given system is variational.

\section{Appendix A. A Direct CALCUlation of SOME integrability CONDItions}

Acting with $\mathrm{D}_{X_{2}}^{V}$ on (2.9) and subtracting the $\mathrm{D}_{X_{2}}^{H}$ prolongation of (2.7), the left-hand side contains the commutator $\left[\mathrm{D}_{X_{2}}^{V}, \mathrm{D}_{X_{2}}^{H}\right]$, for which we make use of the general identity

$$
\left[\mathrm{D}_{X_{i}}^{V}, \mathrm{D}_{X_{j}}^{H}\right]=\mathrm{D}_{\mathrm{D}_{X_{i}}^{V} X_{j}}^{H}-\mathrm{D}_{\mathrm{D}_{X_{j}}^{H} X_{i}}^{V}+\mu_{\theta\left(X_{i}, X_{j}\right)} .
$$

The last term on the right represents an algebraic type derivation and in fact has zero action on functions, but we will use the property also on the $(0,2)$ tensors $g^{A}$ in a moment. So for the immediate need, making use of some relations (2.1), we have

$$
\left[\mathrm{D}_{X_{2}}^{V}, \mathrm{D}_{X_{2}}^{H}\right] \rho_{1}=\tau_{22}^{k} \mathrm{D}_{X_{k}}^{H}-\mu_{22}^{k} \mathrm{D}_{X_{k}}^{V} .
$$

Rearranging the resulting integrability requirement to isolate the highest-order derivative terms in the left-hand side, we obtain

$$
\begin{aligned}
& \left(\mathrm{D}_{X_{2}}^{V} \mathrm{D}_{X_{1}}^{H}-\mathrm{D}_{X_{2}}^{H} \mathrm{D}_{X_{1}}^{V}\right) \rho=\tau_{22}^{k} \mathrm{D}_{X_{k}}^{H} \rho_{1}-\mu_{22}^{k} \mathrm{D}_{X_{k}}^{V} \rho_{1}-\left(\mathrm{D}_{X_{2}}^{V} \rho_{A}\right)\left(\kappa_{13}^{A}-\kappa_{21}^{A}\right) \\
& \quad+\left(\mathrm{D}_{X_{2}}^{H} \rho_{A}\right)\left(v_{13}^{A}-v_{21}^{A}\right)-\rho_{A}\left(\mathrm{D}_{X_{2}}^{V} \kappa_{13}^{A}-\mathrm{D}_{X_{2}}^{H} v_{13}^{A}-\mathrm{D}_{X_{2}}^{V} \kappa_{21}^{A}+\mathrm{D}_{X_{2}}^{H} v_{21}^{A}\right)
\end{aligned}
$$


Repeating the same process for the computation of $\mathrm{D}_{X_{1}}^{V}(2.10)-\mathrm{D}_{X_{1}}^{H}(2.8)$, we find, after interchanging also the second-order derivatives acting on $\rho$, the similar condition

$$
\begin{aligned}
& \left(\mathrm{D}_{X_{2}}^{V} \mathrm{D}_{X_{1}}^{H}-\mathrm{D}_{X_{2}}^{H} \mathrm{D}_{X_{1}}^{V}\right) \rho=-\tau_{11}^{k} \mathrm{D}_{X_{k}}^{H} \rho_{2}+\mu_{11}^{k} \mathrm{D}_{X_{k}}^{V} \rho_{2}+\tau_{12}^{k} \mathrm{D}_{X_{k}}^{H} \rho-\mu_{12}^{k} \mathrm{D}_{X_{k}}^{V} \rho \\
& \quad+\left(\mathrm{D}_{X_{1}}^{V} \rho_{A}\right)\left(\kappa_{23}^{A}-\kappa_{12}^{A}\right)+\tau_{21}^{k} \mathrm{D}_{X_{k}}^{H} \rho-\mu_{21}^{k} \mathrm{D}_{X_{k}}^{V} \rho-\left(\mathrm{D}_{X_{1}}^{H} \rho_{A}\right)\left(v_{23}^{A}-v_{12}^{A}\right) \\
& \quad+\rho_{A}\left(\mathrm{D}_{X_{1}}^{V} \kappa_{23}^{A}-\mathrm{D}_{X_{1}}^{H} v_{23}^{A}-\mathrm{D}_{X_{1}}^{V} \kappa_{12}^{A}+\mathrm{D}_{X_{1}}^{H} v_{12}^{A}\right)
\end{aligned}
$$

The two second-order equations thus obtained are not yet written in their appropriate form. Following the general principles of the Riquier theory indeed, we still have to make some substitutions from the closure conditions $(2.7-2.10)$ in the right-hand sides. Having done that, we propose to show that the difference between the right-hand sides of A.2 and A.3 is identically zero. That the coefficients of each of the remaining first-order derivative terms in the $\rho_{A}$ cancel out is a matter of straight verification, using where necessary the explicit expressions (2.6) for the $v_{i B}^{A}$ and $\kappa_{i B}^{A}$ in terms of the structure functions $\tau_{i j}^{k}, \mu_{i j}^{k}$. Verifying that the same is true for the remaining algebraic terms is more involved, because some of these terms contain in their coefficients horizontal and vertical derivatives of the $v_{i B}^{A}$ and $\kappa_{i B}^{A}$. Recalling that all structure functions are known in principle, we should make use of certain identities that their derivatives will satisfy. At the level of the structure functions of the $g^{A}$ in (2.5), such identities follow for example from expressing the equality A.1 in its action on the $g^{A}$. This gives rise to the following general properties:

$$
\begin{aligned}
& \left(\mathrm{D}_{X_{i}}^{V} \kappa_{j B}^{A}-\mathrm{D}_{X_{j}}^{H} v_{i B}^{A}+\kappa_{j C}^{A} v_{i B}^{C}-v_{i C}^{A} \kappa_{j B}^{C}\right) g^{B} \\
& \quad=\left(\tau_{i j}^{k} \kappa_{k B}^{A}-\mu_{j i}^{k} v_{k B}^{A}\right) g^{B}-g^{A}\left(\theta\left(X_{i}, X_{j}\right) \cdot, \cdot\right)-g^{A}\left(\cdot, \theta\left(X_{i}, X_{j}\right) \cdot\right) .
\end{aligned}
$$

Evaluating this tensor equality for example on the arguments $\left(X_{1}, X_{2}\right)$ with the choice $i=2, j=1$, it is clear that we will obtain an expression for $\mathrm{D}_{X_{2}}^{V} \kappa_{13}^{A}-\mathrm{D}_{X_{1}}^{H} v_{23}^{A}$. Doing the same with $i=1, j=2$, we find an expression for $\mathrm{D}_{X_{1}}^{V} \kappa_{23}^{A}-\mathrm{D}_{X_{2}}^{H} v_{13}^{A}$. With $i=j=1$, the $\left(X_{2}, X_{2}\right)$ component yields an expression for $\mathrm{D}_{X_{1}}^{V} \kappa_{12}^{A}-\mathrm{D}_{X_{1}}^{H} v_{12}^{A}$. Finally, with $i=j=2$, the $\left(X_{1}, X_{1}\right)$ component tells us what to substitute for $\mathrm{D}_{X_{2}}^{V} \kappa_{21}^{A}-\mathrm{D}_{X_{2}}^{H} v_{21}^{A}$. This way, we have expressions for all terms of this type coming into the difference between (A.2) and (A.3). Observe that after these substitutions we will have introduced eight terms of the type $g^{A}\left(\theta\left(X_{i}, X_{j}\right) X_{k}, X_{l}\right)$; immediately, however, they pairwise cancel out in view of the complete symmetry of $\theta$. It is then straightforward, though still quite tedious, to verify, using the expressions (2.6) at the final stage, that all terms cancel out.

This may seem like a very complicated calculation to carry out, but we must not forget that we have obtained after all a quite general result. Indeed, as mentioned at the start of Section 2 and shown explicitly in Sections 3 and 4, there are relations also between the $\mu_{i j}^{k}$ and the $\tau_{i j}^{k}$, which are different for each subcase at hand, because they depend on the freedom left in $g$ after imposing the algebraic conditions, and sometimes also on some extra assumptions. But we have not needed any such properties to find that all terms cancel out. Therefore, we have shown that, in all cases where choosing principal derivatives as in (2.7.2.10 makes sense (this includes all cases where $g$ will be diagonal with $\rho_{1} \neq \rho_{2}$ ), the two direct integrability conditions which could be computed from the closure conditions will coincide. Such 
a result was obtained by Douglas by doing the computation for each such case separately.

\section{REFERENCES}

[1] I. Anderson and G. Thompson, The inverse problem of the calculus of variations for ordinary differential equations, Memoirs Amer. Math. Soc. 98 No. 473 (1992). MR 92k:58070

[2] M. Crampin, E. Martínez and W. Sarlet, Linear connections for systems of second-order ordinary differential equations, Ann. Inst. H. Poincaré Phys. Théor. 65 (1996), 223-249. MR 97g:58003

[3] M. Crampin, G.E. Prince, W. Sarlet and G. Thompson, The inverse problem of the calculus of variations: separable systems, Acta Appl. Math. 57 (1999), 239-254. MR 2001j:58031

[4] M. Crampin, W. Sarlet, E. Martínez, G.B. Byrnes and G.E. Prince, Towards a geometrical understanding of Douglas's solution of the inverse problem of the calculus of variations, Inverse Problems 10 (1994), 245-260. MR 95e:49042

[5] J. Douglas, Solution of the inverse problem of the calculus of variations, Trans. Amer. Math. Soc. 50 (1941), 71-128. MR 3:54c

[6] J. Grifone and Z. Muzsnay, Sur le problème inverse du calcul des variations: existence de lagrangiens associées à un spray dans le cas isotrope, Ann. Inst. Fourier 49 (1999), 1-35. MR 2000d:49035

[7] E. Martínez, J.F. Cariñena and W. Sarlet, Derivations of differential forms along the tangent bundle projection, Differential Geometry and its Applications 2 (1992), 17-43. MR 94h:58004

[8] E. Martínez, J.F. Cariñena and W. Sarlet, Derivations of differential forms along the tangent bundle projection. Part II, Differential Geometry and its Applications 3 (1993), 1-29. MR 94h:58005

[9] E. Martínez, J.F. Cariñena and W. Sarlet, Geometric characterization of separable secondorder differential equations, Math. Proc. Cambridge Philos. Soc. 113 (1993), 205-224. MR 93m:58007

[10] Z. Muzsnay, Sur le problème inverse du calcul des variations, Thèse de Doctorat, Université Paul Sabatier (Toulouse III) (1997).

[11] C. Riquier, Les systèmes d'équations aux derivées partielles, (Gauthier-Villars, Paris) (1910).

[12] W. Sarlet and M. Crampin, Addendum to: The integrability conditions in the inverse problem of the calculus of variations for second-order ordinary differential equations, Acta Appl. Math. 60 (2000), 213-224. MR 2001e:58020

[13] W. Sarlet, M. Crampin and E. Martínez, The integrability conditions in the inverse problem of the calculus of variations for second-order ordinary differential equations, Acta Appl. Math. 54 (1998), 233-273. MR 99m:58072

[14] W. Sarlet, A. Vandecasteele, F. Cantrijn and E. Martínez, Derivations of forms along a map: The framework for time-dependent second-order equations, Differential Geometry and its Applications 5 (1995), 171-203. MR 96m:58006

Department of Mathematical Physics and Astronomy, Ghent University, Krijgslaan 281, B-9000 Ghent, Belgium

E-mail address: willy.sarlet@rug.ac.be

Department of Mathematics, The University of Toledo, Toledo, Ohio 43606

E-mail address: gthomps@uoft02.utoledo.edu

School of Mathematics, University of New South Wales, Sydney NSW 2052, AusTRALIA

Current address: Department of Mathematics, La Trobe University, Victoria 3086, Australia

E-mail address: g.prince@latrobe.edu.au 